\title{
THE CENTER OF THE GENERIC G-CROSSED PRODUCT
}

\author{
OFIR DAVID
}

\begin{abstract}
Let $G$ be a finite group and let $\mathbb{F}$ be a field of characteristic zero. In this paper we construct a generic $G$-crossed product over $\mathbb{F}$ using generic graded matrices. The center of this generic $G$-crossed product, denoted by $\mathbb{F}(G)$, is then the invariant field of a suitable $G$ action on a field of rational functions in several indeterminates. The main goal of this paper is to study the extensions $\mathbb{F}(G) / \mathbb{F}$ given that $\mathbb{F}$ contains enough roots of unity and determine how close they are to being purely transcendental.

In particular we show that $\mathbb{F}(G) / \mathbb{F}$ is a stably rational extension for $G=C_{2} \times C_{2 n}$ where $n$ is odd and for $G=\left\langle\sigma, \tau \mid \sigma^{n}=\tau^{2 m}=e, \tau \sigma \tau^{-1}=\sigma^{-1}\right\rangle$ where $\operatorname{gcd}(n, 2 m)=1$. Furthermore, we prove that if $H, K$ are groups of coprime orders, then $\mathbb{F}(H \times K)$ is the fraction field of $\mathbb{F}(H) \times \mathbb{F}(K)$.
\end{abstract}

\section{INTRODUCTION}

The Brauer group $B r(\mathbb{F})$ of a field $\mathbb{F}$ is considered as one of its most important arithmetic invariants. It consists of all the finite dimensional division algebras central over $\mathbb{F}$ (or equivalently, central simple algebras up to Morita equivalence). An important tool used in studying this group, and more generally the groups $B r(\mathbb{L})$ for field extensions $\mathbb{L} / \mathbb{F}$, is the generic division algebra $D_{n}$ of degree $n$ over the field $\mathbb{F}$. It has the remarkable property that any other central simple algebra of degree $n$ of a field extension of $\mathbb{F}$ is a specialization of $D_{n}$. The generic algebra's usefulness comes mainly from the fact that many nice properties satisfied by it are inherited by all of its specializations (see [32] for details). For example, if $D_{n}$ is a crossed product with a group $G$, then any division algebra of degree $n$ over a field extension of $\mathbb{F}$ is also a crossed product with the same group $G$. Amitsur used this property to show that for suitable integers $n$ the generic division algebra $D_{n}$ is not a crossed product. This was the first example found of a noncrossed product division algebra (see [4]).

Let us recall another such property. A central simple algebra is called cyclic if it is isomorphic to a crossed product with a cyclic group. The Merkurjev-Suslin theorem [24] states that over a field $\mathbb{F}$ containing enough roots of unity, every central simple algebra is equivalent to a tensor product of cyclic algebras, or in other words $B r(\mathbb{F})$ is generated by the equivalence classes of cyclic algebras. While Merkurjev-Suslin's proof uses K-cohomology, another approach to this problem, given prior to their proof, uses generic algebras. It is not difficult to show that if $D_{n}$ is equivalent to a product of cyclic algebras, then so is every central simple algebra of degree $n$ over a field extension of $\mathbb{F}$. Consider an algebraic field extension $\mathbb{F}$ of $\mathbb{Q}$ containing all roots of unity. Then it is known that $\operatorname{Br}(\mathbb{F})$ is trivial, namely the only central simple algebras over it are matrix algebras, and in particular $\operatorname{Br}(\mathbb{F})$ is generated by classes of cyclic algebras (in a trivial way). Furthermore, by a well known theorem due to Bloch, the property of generation by classes of cyclic algebras extends to $\operatorname{Br}\left(\mathbb{F}\left(x_{1}, \ldots, x_{n}\right)\right)$ whenever the $x_{i}$ are algebraically independent over $\mathbb{F}$. Using these ideas, if one could show that the center of $D_{n}$ is a rational extension of $\mathbb{F}$, then it will follow that any central simple algebra of degree

Key words and phrases. generic crossed products, G-lattices, stably rational extension.

The author was supported by THE ISRAEL SCIENCE FOUNDATION (grant No. 1017/12). 
$n$ over a field extension of $\mathbb{F}$ is equivalent to a product of cyclic algebras, thus proving MerkurjevSuslin's theorem for such fields (for more details and a proof of Bloch's theorem using the Auslander Brumer Faddeev theorem see [12], and for Bloch's original proof see [9]).

The last argument demonstrates the significance of the center $Z_{n}:=Z\left(D_{n}\right)$ of $D_{n}$ in the study of Brauer groups. In particular, we are interested in determining if the field extension $Z_{n} / \mathbb{F}$ is rational (i.e. purely transcendental), or has some weaker rationality property, namely it is stably rational, retract rational or just unirational (the definitions for the different types of rationality extensions are given in Section 3).

Over the rational field $\mathbb{Q}$, the first result in this direction is attributed to Sylvester, who showed in 1883 that $Z_{2} / \mathbb{Q}$ is rational (see [36]). About 80 years later, using the algebra of generic matrices to construct the generic algebra, Procesi was able to show that $Z_{n}$ is the invariant field of a suitable $S_{n}$ action on a rational extension of $\mathbb{Q}$, hence in particular these field extensions are unirational (see [27]). Applying Procesi's method, Formanek proved the rationality for $n=3,4$ (see [14, 15] and also [16]). Le Bruyn and Bessenrodt in [8] proved that for $n=5,7$ the field $Z_{n}$ is a stably rational extension of $\mathbb{Q}$ and Beneish in [7] gave a more elementary proof for these primes. Schofield [33], Katsylo [19] and Saltman [31] showed that $Z_{n m}$ is stably rationally equivalent to the fraction field of $Z_{n} \otimes Z_{m}$ whenever $n, m$ are coprime, thus reducing the problem to the prime power case. Finally, Saltman showed in [30] that $Z_{p}$ is retract rational over $\mathbb{F}$ for $p$ prime, and this result can be extended to product of distinct primes. For more information see Le Bruyn's survey [21].

It is well known that any central simple algebra is Brauer equivalent to a $G$-crossed product for some finite group $G$, hence it is only natural to consider generic $G$-crossed products. Indeed, several equivalent constructions were given by Snider [34] and Rosset [28], who used relation modules, and by Saltman [32, 30], who used generic 2-cocycles. As with the generic algebra case, many properties of the generic crossed product are inherited to all the $G$-crossed products, and in particular the idea mentioned above for proving the Merkurjev-Suslin theorem is still applicable here (see for example $[34])$.

With this goal in mind, Snider proved that the center of the generic $G$-crossed product is a rational extension over $\mathbb{Q}$ where $G$ is the Klein four group and stably rational for Dihedral groups of order $2 n$ over $\mathbb{Q}\left(\zeta_{2 n}\right)$ where $\zeta_{2 n}$ is a primitive $2 n$-root of unity. Another result due to Saltman shows that if all the $p$-Sylow subgroups of $G$ are cyclic then the center of the generic $G$-crossed product is retract rational over a suitable extension of the base field. Recall that a group $G$ such that all of its $p$-Sylow subgroups are cyclic is a semidirect product of cyclic groups $C_{n} \rtimes C_{m}$ where $\operatorname{gcd}(n, m)=1$ (see [17], section 9.4). This family contains the affine groups $\mathbb{F}_{q} \rtimes \mathbb{F}_{q}^{\times}$of finite fields which appear in Beneish's work, where she proves that the centers of the generic division algebras of rank 5 and 7 are stably rational extensions over $\mathbb{Q}$. On the other hand, Saltman proved that roughly speaking these are almost all the groups for which the center may be close to being a rational extension. More precisely, Saltman showed that the center can be retract rational only if each $p$-Sylow subgroup is either cyclic or a product of two cyclic groups.

The goal of this paper is to further study the centers of the generic crossed products. For a group $G$ and a field $\mathbb{F}$, denote by $\mathbb{F}(G)$ the center of the generic $G$-crossed product over the field $\mathbb{F}$. In what follows, we always assume that $\mathbb{F}$ contains a primitive root of unity of order $|G|$. The first result deals with groups containing only cyclic $p$-Sylow subgroups.

Theorem 1.1. Let $G=C_{n} \rtimes C_{m}, \operatorname{gcd}(m, n)=1$, be a group with cyclic $p$-Sylow subgroup. Then:

(1) The extension $\mathbb{F}(G) / \mathbb{F}$ is retract rational.

(2) If $K=Z(G) \cap C_{m}$ is the kernel of the action of $C_{m}$ on $C_{n}$ by conjugation, then the extension $\mathbb{F}(G) / \mathbb{F}$ is stably isomorphic to $\mathbb{F}(G / K) / \mathbb{F}$. 
Combining Snider's result for Dihedral groups and part (2) of the theorem above we conclude the following.

Corollary 1.2. Let. $G=\left\langle\sigma, \tau \mid \sigma^{n}=\tau^{2 m}=e, \tau \sigma \tau^{-1}=\sigma^{-1}\right\rangle$ where $\operatorname{gcd}(n, 2 m)=1$. Then $\mathbb{F}(G) / \mathbb{F}$ is a stably rational extension.

Recall that for the standard generic division algebra, the question of rationality can be reduced to the prime power case. The next result is its counterpart for generic crossed products.

Theorem 1.3. Let $G \cong H \times K$ where $H, K$ are groups of coprime orders. Then $\mathbb{F}(G) / \mathbb{F}$ is stably rationally equivalent to the fraction field of $\mathbb{F}(H) \otimes \mathbb{F}(K)$.

Using Snider's result for the Klein four group we conclude the following.

Corollary 1.4. The extension $\mathbb{F}\left(C_{2} \times C_{2 n}\right) / \mathbb{F}$ is stably rational whenever $n$ is odd and $\mathbb{F}$ contains a primitive $n$-th root of unity.

Interestingly, it is still unknown whether $\mathbb{F}(G) / \mathbb{F}$ is stably rational for $G=C_{p} \times C_{p}$ with $p \geq 3$ prime.

The paper is organized as follows.

We start with the construction of the generic crossed product in Section 2 using generic graded matrices and give a description of its center using $G$-lattices. In Section 3 we recall the definitions and main results needed from the theory of $G$-lattices and their field invariants.

In Section 4 we introduce the concept of flows in graphs and use them in order to construct the $G$ lattices appearing both in study of center of the generic division algebra and generic crossed product. The main results of this paper are proved in Section 5 where we restrict to the flows representing the center of generic crossed products. Finally, in Section 6 we recall some results on the center of the standard generic division algebra and interpret them using the language of flows in graphs.

\section{Generic Crossed Products}

Fix a field $\mathbb{F}$ of characteristic zero. All the rings in this section are algebras over $\mathbb{F}$, and the homomorphism are always $\mathbb{F}$-homomorphisms.

The generic division algebra and its center have been extensively studied in the literature. A detailed account can be found in [16] and in Le Bruyn's survey [21] which details the motivation and different approaches to study the center of these generic algebras. An analogous object can be defined for the class of $G$-crossed products for a fixed finite group $G$. It has several equivalent constructions in the literature using relation modules by Snider and Rosset [34, 28] and generic 2-cocycle by Saltman $[30,32]$. A more general approach using graded polynomial identities was studied by Aljadeff and Karasik [1]. The polynomial identities construction was first used for the standard generic division algebra, and can be further generalized to generic algebras for other classes of graded simple algebra (which include crossed product) and even to generic Hopf algebras [3]. The approach utilized here uses generic graded matrices which is very similar to the one with graded polynomial identities.

Usually, $G$-crossed products are defined over Galois extensions of fields. For the generic crossed product definition we require the more generalized definition of Galois extensions of rings, which we briefly describe. For a full treatment of Galois extension of commutative rings and the crossed products defined over them, we refer the reader to [25] and [26]. 
Definition 2.1 (Galois Extension). Let $R \subseteq S$ be an extension of unital commutative rings, and let $G$ be a finite subgroup of $\operatorname{Aut}(S)$. We say that $S$ is a $G$-Galois extension of $R$ if

(1) $S$ is a faithful $R$ algebra.

(2) $S^{G}=R$.

(3) There are $x_{1}, \ldots, x_{n}, y_{1}, \ldots, y_{n} \in S$ for some $n \in \mathbb{N}$ such that $\sum_{i} x_{i} g\left(y_{i}\right)=\delta_{e, g}$ where $\delta_{e, g}=1$ if $g=e$ and zero otherwise.

Definition 2.2 (G-Crossed Product). Let $S$ be a $G$-Galois extension of $R$ and let $c \in Z^{2}\left(G, S^{\times}\right)$be a 2-cocycle, namely $c$ is a function from $G \times G$ to $S^{\times}$that satisfies

$$
\text { for all } g_{1}, g_{2}, g_{3} \in G \quad c\left(g_{1}, g_{2}\right) c\left(g_{1} g_{2}, g_{3}\right)=g_{1}\left(c\left(g_{2}, g_{3}\right)\right) c\left(g_{1}, g_{2} g_{3}\right) .
$$

Consider the $R$-module $\bigoplus_{g \in G} S \cdot \epsilon_{g}$ with a multiplication defined by

$$
\text { for all } \alpha, \beta \in S, g, h \in G \quad \alpha \epsilon_{g} \beta \epsilon_{h}=\alpha g(\beta) \epsilon_{g} \epsilon_{h}=\alpha g(\beta) c(g, h) \epsilon_{g h} .
$$

This algebra is called a $G$-crossed product and is denoted by $\Delta(S / R, G, c)$.

Crossed products over Galois extensions of fields are essential in the study of central simple algebras. Their generalized versions play a similar role in the study of Azumaya algebras and have many similar properties. In particular, cohomologous 2-cocycles produce isomorphic crossed product, so we can assume that the two cocycles are always normalized, namely $\epsilon_{e}$ is the unity of $\Delta(S / R, G, c)$, and hence we identify $S$ with $S \cdot \epsilon_{e}$ and $R$ with $R \cdot \epsilon_{e}$. Under this notation, the crossed product $\Delta(S / R, G, c)$ is an Azumaya algebra central over $S^{G}=R$.

Recall that a $G$-grading of an $S$-algebra $A$, is a decomposition $A=\bigoplus_{g \in G} A_{g}$ as an $S$-module such that $A_{g} A_{h} \subseteq A_{g h}$. Clearly, setting $\Delta_{g}=S \cdot \epsilon_{g}$ produces a $G$-grading of $\Delta:=\Delta(S / R, G, c)$. It is well known that any crossed product over a Galois extension of fields is central simple, and therefore after suitable scalars extension it becomes a matrix algebra. The crossed product's natural group grading induces a grading on that matrix algebra which we now describe.

Definition 2.3 (Elementary Grading). Let $R$ be an $\mathbb{F}$-algebra, $A=M_{n}(R)$ and $\bar{g}=\left(g_{1}, \ldots, g_{n}\right) \in$ $G^{n}$ be a tuple of length $n$. The elementary grading on $A$ induced by $\bar{g}$ is defined by $A_{h}=$ $\operatorname{span}_{R}\left\{E_{i, j} \mid g_{i}^{-1} g_{j}=h\right\}$, where $E_{i, j}$ is the matrix with 1 in the $(i, j)$ coordinate and zero elsewhere. In case each element of $G$ appears exactly once in $\bar{g}$, the induced elementary grading is called the crossed product grading.

Remark 2.4. Note that reordering the tuple $\bar{g}$ produces graded isomorphic algebras.

Let $M_{n}(R)$ have the crossed product grading with a tuple $\bar{g}=\left(g_{1}, \ldots, g_{n}\right) \in G^{n}$, where $n=|G|$. Identifying $G$ with $\{1, \ldots, n\}$, we write $E_{g_{i}, g_{j}}$ instead of $E_{i, j}$. Let $S$ be the subalgebra of diagonal matrices in $M_{n}(R)$ and set $P_{g}$ to be the permutation matrix $P_{g}:=\sum_{h \in G} E_{h, h g}$. These permutation matrices play the roles of $\epsilon_{g}$ in the definition of $G$-crossed products, and since $P_{g} P_{h}=P_{g h}$, the corresponding two cocycle is trivial. Letting $G$ act on $S$ by $g(s)=P_{g} s P_{g}^{-1}$ for $g \in G$ and $s \in S$ we get that $S^{G} \cong R$ are the scalar matrices and $S / S^{G}$ is a $G$-Galois extension and therefore $M_{n}(R) \cong$ $\Delta(S / R, G, 1)$ with $\left(M_{n}(R)\right)_{g}=S \cdot P_{g}$. Unless otherwise stated, we will always assume that the grading on $M_{n}(R)$ is the crossed product grading. 
Theorem 2.5. If $\Delta=\Delta(S / R, G, c)$ is a G-crossed product where $R$ is an integral domain, then there is some field $R \subseteq \mathbb{L}$ such that $\Delta \otimes_{R} \mathbb{L} \cong M_{|G|}(\mathbb{L})$ as graded algebras where $M_{|G|}(\mathbb{L})$ has the crossed product grading.

Proof. This result is well known, see for example Theorem 10 in [2]. For clarity we give here a sketch of the proof.

First, by extending the scalars by the field of fractions of $R$, we may assume that $R$ is a field. Since $S / R$ is Galois with $R$ a field, we get that $S \cong \mathbb{L}^{k}$ where $\mathbb{L} / R$ is a field extension and $[\mathbb{L}: R] \cdot k=|G|$. Thus, extending the scalars by $\mathbb{L}$, we get the Galois extension $\mathbb{L}^{n} / \mathbb{L}$ where the group $G$ acts on $\mathbb{L}^{n}$ by permuting the idempotents via the regular representation of $G$.

Since $\mathbb{L}$ is a field and $\Delta_{\mathbb{L}}:=\Delta \otimes_{R} \mathbb{L}$ is central simple, we have an isomorphism $\Delta_{\mathbb{L}} \cong M_{|G|}(\mathbb{L})$. Secondly, since $S \cong \mathbb{L}^{k}$, we may assume (after another autmorphism) that $S$ is the set of diagonal matrices and $R$ is the set of scalar matrices. Under this identification, the elements $\epsilon_{g} P_{g}^{-1}$ commute with the diagonal matrices, and therefore must be diagonal, so that $\epsilon_{g}=t_{g} P_{g}$ for some $t_{g}$ invertible and diagonal. It follows that $\Delta_{g}=S \epsilon_{g}=S \cdot P_{g}$ is exactly the crossed product grading.

With the crossed product grading in mind, we turn to construct the generic $G$-crossed product. Define a $g$-generic matrix to be

$$
X_{g, i}=\left(\begin{array}{cccc}
x_{g_{1}, g_{1} g}^{(i)} & 0 & \cdots & 0 \\
0 & x_{g_{2}, g_{2} g}^{(i)} & & \vdots \\
\vdots & & \ddots & 0 \\
0 & \cdots & 0 & x_{g_{n}, g_{n} g}^{(i)}
\end{array}\right) P_{g},
$$

which is homogeneous of degree $g$ in $M_{|G|}\left(\mathbb{F}\left(x_{h, g}^{(i)}\right)\right)$ with the crossed product grading, where the $x_{h, g}^{(i)}$ are algebraically independent over $\mathbb{F}$. Let $R_{G, k}$ be the unital $\mathbb{F}$-subalgebra of $M_{|G|}\left(\mathbb{F}\left(x_{h, g}^{(i)}\right)\right)$ generated by $\left\{X_{g, i} \mid g \in G, 1 \leq i \leq k\right\}$. The generic crossed product $D_{G, k}$ is $R_{G, k}$ after inverting all the nonzero central elements.

Next, we give a presentation of the $e$ component of $D_{G, k}$ as a Galois field extension of the center of $D_{G, k}$.

Theorem 2.6. Let $\mathbb{L} \leq \mathbb{F}\left(x_{g, h}^{(i)}\right)$ be the field extension of $\mathbb{F}$ generated by elements of the form

$$
x_{h, h g_{1}}^{\left(j_{1}\right)} x_{h g_{1}, h g_{1} g_{2}}^{\left(j_{2}\right)} x_{h g_{1} g_{2}, h g_{1} g_{2} g_{3}}^{\left(j_{3}\right)} \cdots x_{h \prod_{1}^{m-1} g_{i}, h \prod_{1}^{m} g_{i}}^{\left(j_{m}\right)},
$$

where $m, j_{1}, \ldots, j_{m} \in \mathbb{N}, h, g_{1}, \ldots, g_{m} \in G$ and $g_{1} \cdots g_{m}=e$. Then $\left(D_{G, k}\right)_{e} \cong \mathbb{L}$ and $Z\left(D_{G, k}\right) \cong \mathbb{L}^{G}$ where the $G$-action is defined by $\sigma\left(x_{h, g}^{(i)}\right)=x_{\sigma h, \sigma g}^{(i)}$.

Proof. In order to study the center $Z\left(R_{G, k}\right)$, note first that these elements are scalar matrices, and in particular homogeneous of degree $e$ (or equivalently diagonal). If $A=\prod X_{g_{i}, j_{i}}$ is a monomial with $\prod g_{i}=e$, then

$$
\begin{aligned}
A_{(h, h)} & =x_{h, h g_{1}}^{\left(j_{1}\right)} x_{h g_{1}, h g_{1} g_{2}}^{\left(j_{2}\right)} x_{h g_{1} g_{2}, h g_{1} g_{2} g_{3}}^{\left(j_{2}\right)} \cdots x_{h \prod_{1}^{m-1} g_{i}, h \prod_{1}^{m} g_{i}}^{\left(j_{m}\right)} \\
& =h\left(x_{e, g_{1}}^{\left(j_{1}\right)} x_{g_{1}, g_{1} g_{2}}^{\left(j_{2}\right)} x_{g_{1} g_{2}, g_{1} g_{2} g_{3}}^{\left(j_{3}\right)} \cdots x_{\prod_{1}^{m-1} g_{i}, \prod_{1}^{m} g_{i}}^{\left(j_{m}\right)}\right)=h\left(A_{(e, e)}\right)
\end{aligned}
$$

for any $h \in H$. Since $\left(R_{G, k}\right)_{e}$ is spanned by such monomials, it follows that $A_{(h, h)}=h\left(A_{(e, e)}\right)$ for any $A \in\left(R_{G, k}\right)_{e}$. Consequently, if $A \in\left(R_{G, k}\right)_{e}$ is nonzero, then all its entries on the diagonal are nonzero, and in particular $\left(R_{G, k}\right)_{e}$ and $Z\left(R_{G, k}\right)$ are integral domains. 
Define $\varphi:\left(R_{G, k}\right)_{e} \rightarrow \mathbb{F}\left(x_{h, g}^{(i)}\right)$ by sending a diagonal matrix to its $(e, e)$ entry. By the preceding argument this map is injective and contains all the elements of the form $x_{e, g_{1}}^{\left(j_{1}\right)} x_{g_{1}, g_{1} g_{2}}^{\left(j_{2}\right)} x_{g_{1} g_{2}, g_{1} g_{2} g_{3}}^{\left(j_{3}\right)} \cdots x_{\prod_{1}^{m-1} g_{i}, \prod_{1}^{m} g_{i}}^{\left(j_{m}\right)}$ and note that $\mathbb{L}$ is exactly the fraction field of $\varphi\left(\left(R_{G, k}\right)_{e}\right)$. Additionally an element is central in $R_{G, k}$ if and only if its image under $\varphi$ is $G$-invariant.

Extend $\varphi$ to $\left(D_{G, k}\right)_{e}$ in the natural way. By definition $\varphi\left(\left(R_{G, k}\right)_{e}\right) \subseteq \varphi\left(\left(D_{G, k}\right)_{e}\right) \subseteq \mathbb{L}$, hence the theorem will be proven if we can show that $\left(D_{G, k}\right)_{e}$ is a field. On the other hand, $Z\left(D_{G, k}\right)$ is a field, so it is enough to show that $\left(D_{G, k}\right)_{e}$ is finite dimensional over it (since it is an integral domain).

The same argument as in the beginning of the proof shows that all the nonzero homogeneous elements in $D_{G, k}$ are invertible in $M_{|G|}\left(\mathbb{F}\left(x_{h, g}^{(i)}\right)\right)$. Recall that a graded algebra $B$ is called graded prime if for any homogeneous elements $b_{1}, b_{2} \in B$ we have $b_{1} B b_{2}=0$ if and only if $b_{1}=0$ or $b_{2}=0$. In particular $D_{G, k}$ is graded prime and by a graded analog of Posner's theorem given in [6], it is graded simple over its center. In particular $D_{G, k} \otimes_{Z\left(D_{G, k}\right)} \mathbb{F}\left(x_{h, g}^{(i)}\right)$ is graded simple and therefore the homomorphism $D_{G, k} \otimes_{Z\left(D_{G, k}\right)} \mathbb{F}\left(x_{h, g}^{(i)}\right) \rightarrow M_{n}\left(\mathbb{F}\left(x_{h, g}^{(i)}\right)\right)$ is injective and can be easily seen to be an isomorphism. It follows that $\operatorname{dim}_{Z\left(D_{G, k}\right)}\left(\left(D_{G, k}\right)_{e}\right)=\operatorname{dim}_{\mathbb{F}\left(x_{h, g}^{(i)}\right)}\left(\left(M_{n}\left(\mathbb{F}\left(x_{h, g}^{(i)}\right)\right)\right)_{e}\right)=n$ is finite, hence $\left(D_{G, k}\right)_{e}$ is indeed a field, and therefore isomorphic to $\mathbb{L}$.

Note that the elements generating the field $\mathbb{L}$ in the last theorem correspond to "cycles" on a Cayley graph for the group $G$. In Section 4 this notion will be formalized and generalized to other graphs, but first we recall some definition and results required for this study.

\section{G-LATtiCES AND FIELD INVARIANTS}

Fix a finite group $G$ and a field $\mathbb{F}$ of characteristic zero with a $G$-action (possibly trivial). In this section we recall the basic definitions and results regarding $G$-lattices and their corresponding field invariants needed for this paper. Further details and proofs can be found in [23] and [20].

Definition 3.1. Let $\mathbb{L} / \mathbb{F}$ be a finitely generated field extension. Then:

- $\mathbb{L}$ is called a rational extension of $\mathbb{F}$ if $\mathbb{L}=\mathbb{F}\left(x_{1}, \ldots, x_{n}\right)$ for some algebraically independent indeterminates $\left\{x_{1}, \ldots, x_{n}\right\}$.

- $\mathbb{L}$ is called a stably rational extension of $\mathbb{F}$ if $\mathbb{L}\left(y_{1}, \ldots, y_{m}\right)$ is rational over $\mathbb{F}$ for some $\left\{y_{1}, \ldots, y_{m}\right\}$ algebraically independent over $\mathbb{L}$.

- $\mathbb{L}$ is called retract rational extension of $\mathbb{F}$ if $\mathbb{L}$ is the fraction field of some $\mathbb{F}$-algebra $A$, and there are homomorphisms $\iota: A \rightarrow \mathbb{F}\left[x_{1}, \ldots, x_{n}\right]\left[s^{-1}\right]$ and $\pi: \mathbb{F}\left[x_{1}, \ldots, x_{n}\right]\left[s^{-1}\right] \rightarrow A$ such that $\pi \circ \iota=i d_{A}$.

- $\mathbb{L}$ is called a unirational extension of $\mathbb{F}$ if $\mathbb{L}$ can be embedded in a rational extension of $\mathbb{F}$.

We always have

rational $\subseteq$ stably rational $\subseteq$ retract rational $\subseteq$ unirational.

We remark here that the inclusions above are all proper, although the examples are not trivial.

Definition 3.2. Two finitely generated field extensions $\mathbb{L}_{1}, \mathbb{L}_{2} / \mathbb{F}$ are called stably isomorphic (over $\mathbb{F})$ if there are $x_{1}, \ldots, x_{m}$ and $y_{1}, \ldots, y_{k}$ algebraically independent over $\mathbb{L}_{1}, \mathbb{L}_{2}$ respectively such that $\mathbb{L}_{1}\left(x_{1}, \ldots, x_{m}\right) \cong \mathbb{L}_{2}\left(y_{1}, \ldots, y_{k}\right)$ as $\mathbb{F}$-algebras. In this case we write $\mathbb{L}_{1} \approx_{\mathbb{F}} \mathbb{L}_{2}$ or just $\mathbb{L}_{1} \approx \mathbb{L}_{2}$ if there is no ambiguity. 
A $G$-lattice $\mathrm{M}$ is a $G$-module which is finitely generated as an abelian group, namely $M \cong \mathbb{Z}^{n}$ for some $n \in \mathbb{N}$. We denote by $\mathbb{F}[M]$ the group algebra of $M$ which is isomorphic to the algebra of Laurent polynomials in $\operatorname{rank}(M)=n$ variables. The $G$-action on $M$ and $\mathbb{F}$ induce a $G$-action on $\mathbb{F}[M]$ by $g\left(\alpha x^{m}\right)=g(\alpha) x^{g(m)}$ for any $g \in G, \alpha \in \mathbb{F}$ and $m \in M$. This action is extended naturally to the field of fractions $\mathbb{F}(M)$ of $\mathbb{F}[M]$. The main question is to find out how close is the extension $\mathbb{F}(M)^{G} / \mathbb{F}^{G}$ to being rational.

A $G$-lattice $M$ is called a permutation lattice if it has a $G$-stable $\mathbb{Z}$ basis $X$, in which case we write $M=\mathbb{Z} X$. Since each $G$-set $X$ is a disjoint union of sets of the form $G / H$ for some subgroup $H \leq G$, it follows that $\mathbb{Z} X \cong \bigoplus_{1}^{t} \mathbb{Z} G / H_{i}$. Notice that if $X=\left\{x_{1}, \ldots, x_{n}\right\}$, then $\mathbb{F}(\mathbb{Z} X)=\mathbb{F}\left(y_{x_{1}}, \ldots, y_{x_{n}}\right)$ where the $y_{x_{i}}$ are algebraically independent over $\mathbb{F}$ and $g\left(y_{x}\right)=y_{g(x)}$ for any $x \in X$.

The importance of permutation lattices is given in the next two results.

Proposition 3.3 (Masuda). Let $\mathbb{F}^{\mathbb{F}} / \mathbb{F}^{G}$ be a $G$-Galois extension of fields and $P$ a permutation lattice. Then $\mathbb{F}(P)^{G}$ is a rational extension of $\mathbb{F}^{G}$.

Theorem 3.4. Let $0 \longrightarrow M_{1} \longrightarrow M_{2} \longrightarrow P \longrightarrow 0$ be an exact sequence of $G$-lattices where $P$ is a permutation lattice. Then $\mathbb{F}\left(M_{2}\right)^{G}$ is rational over $\mathbb{F}\left(M_{1}\right)^{G}$ if one of the following conditions is satisfied:

(1) $M_{1}$ is a faithful $G$-lattice and $\mathbb{F}$ has a trivial $G$-action.

(2) $\mathbb{F} / \mathbb{F}^{G}$ is a $G$-Galois extension of fields.

Remark 3.5. Noether's Problem asks if given a field $\mathbb{F}$ with trivial $G$-action and a faithful permutation lattice $M$, how close is $\mathbb{F}(M)^{G} / \mathbb{F}$ to be a rational extension. This problem was studied extensively in the literature and was solved for many families of groups. I particular, a positive solution for this problem implies a positive solution to the inverse Galois problem. For example, the rationality of $\mathbb{F}\left(\mathbb{Z}^{S_{n}} / S_{n-1}\right)^{S_{n}} / \mathbb{F}$ follows from the fundamental theorem of symmetric polynomials and was used by Hilbert to show that $S_{n}$ is realizable as a Galois extension over $\mathbb{Q}$. Noether's problem was also solved completely for abelian groups by Lenstra in [22] for any field $\mathbb{F}$. For the case where $\mathbb{F}$ contains a root of unity of the order of the exponent of $G$ and $G$ is abelian, Fischer showed that $\mathbb{F}(\mathbb{Z} G)^{G} / \mathbb{F}$ is always rational [13]. A generalization of this result where the action of $G$ on $\mathbb{F}$ is not trivial is given in Lemma 5.12. Many cases appearing in this paper are answered by a reduction to Noether's Problem.

A $G$-lattice $M$ is called quasi-permutation if there is an exact sequence $0 \longrightarrow M \longrightarrow Q \longrightarrow P \longrightarrow 0$ where $P$ and $Q$ are permutation lattices. In particular, under the conditions of the last theorem on $M$ and $\mathbb{F}$, we get that $\mathbb{F}(M)^{G} \approx \mathbb{F}(\mathbb{Z} G)^{G}$.

One trivial way to find an exact sequence as in the theorem above is if $M_{2} \cong M_{1} \oplus P$. With this in mind we say that two lattice $M_{1}, M_{2}$ are called permutation equivalent or just equivalent if there are permutation lattices $P_{1}, P_{2}$ such that $M_{1} \oplus P_{1} \cong M_{2} \oplus P_{2}$. In this case we write $M_{1} \sim M_{2}$ and denote the equivalence class by $\left[M_{1}\right]$. It follows, for example, that if $M_{1}, M_{2}$ are faithful $G$-lattices, $\mathbb{F}$ has a trivial $G$-action and $M_{1} \sim M_{2}$, then $\mathbb{F}\left(M_{1}\right)^{G} \approx \mathbb{F}\left(M_{2}\right)^{G}$.

The set of $G$-lattices modulo the relation $\sim$ has the structure of an abelian semi group with the action $\left[M_{1}\right]+\left[M_{2}\right]=\left[M_{1} \oplus M_{2}\right]$. A lattice $M$ is called invertible or permutation projective if $[M]$ is invertible in this semigroup, or equivalently $M$ is a direct summand of a permutation lattice.

For a subgroup $\tilde{G} \leq G$, we denote by $\hat{H}^{i}(\tilde{G}, M)$ the $i$-th Tate cohomology group. Recall that any short exact sequence $0 \rightarrow A \rightarrow B \rightarrow C \rightarrow 0$ defines a long exact series

$$
\cdots \longrightarrow \hat{H}^{-1}(\tilde{G}, C) \longrightarrow \hat{H}^{0}(\tilde{G}, A) \longrightarrow \hat{H}^{0}(\tilde{G}, B) \longrightarrow \hat{H}^{0}(\tilde{G}, C) \longrightarrow \hat{H}^{1}(\tilde{G}, A) \longrightarrow \cdots
$$


A lattice $M$ is called flasque or $\hat{H}^{-1}$-trivial (resp. coflasque or $\hat{H}^{1}$-trivial) if $\hat{H}^{-1}(\tilde{G}, M)=0$ for any subgroup $\tilde{G} \leq G\left(\operatorname{resp} . \hat{H}^{1}(\tilde{G}, M)=0\right)$. Any permutation lattice, and therefore any invertible lattice, is flasque and coflasque, though the converse is not necessarily true.

It is well known that any surjection $M \rightarrow P$ on a projective module $P$ is split. The following is the analog for invertible modules.

Lemma 3.6. Let

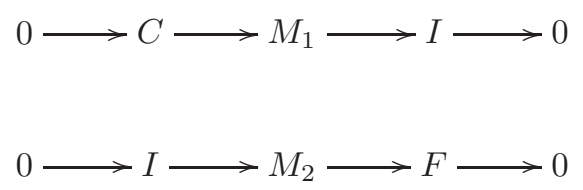

be exact sequences where $I$ is invertible, $C$ is coflasque and $F$ is flasque. Then these sequences split.

A flasque resolution of $M$ is a short exact sequence of the form

$$
0 \longrightarrow M \longrightarrow P \longrightarrow F \longrightarrow 0
$$

where $P$ is a permutation lattice and $F$ is flasque. Similarly a coflasque resolution of $M$ is a short exact sequence of the form

$$
0 \longrightarrow C \longrightarrow P \longrightarrow M \longrightarrow 0
$$

where $P$ is a permutation lattice and $C$ is coflasque. Note in particular that a flasque and coflasque resolutions of an invertible lattice split.

Denote by $M^{*}$ the dual of $M$, namely $M^{*}=\operatorname{Hom}(M, \mathbb{Z})$. It has a natural $G$-structure making it into a $G$-lattice. It follows from the Tate duality that $\hat{H}^{i}(\tilde{G}, M) \cong \hat{H}^{-i}\left(\tilde{G}, M^{*}\right)$ for any lattice $M$, subgroup $\tilde{G} \leq G$ and an integer $i \in \mathbb{Z}$, so in particular $M$ is flasque if and only if $M^{*}$ is coflasque. Since any permutation lattice is self dual, it follows that the dual of a flasque resolution of $M$ is a coflasque resolution of $M^{*}$ and vice versa.

It is well known that any lattice $M$ admits a coflasque (and therefore a flasque) resolution. Moreover, the lattices $F$ and $C$ appearing in the sequences above are unique up to the relation $\sim$ (which is the analog of Schanuel's lemma).

Lemma 3.7. Let $M_{1}, M_{2}$ be lattices and let $F_{1}, F_{2}$ be flasque lattices appearing in flasque resolutions of $M_{1}$ and $M_{2}$ respectively. If $M_{1} \sim M_{2}$, then $F_{1} \sim F_{2}$.

Following this theorem, we write $[M]^{f l}$ to be the equivalence class $[F]$ where $F$ is a flasque lattice appearing in a flasque resolution of $M$. Similarly we write $[M]^{\text {cofl }}$ for coflasque resolutions.

The next result generalizes Theorem 3.4.

Theorem 3.8. Let $M_{1}, M_{2}$ be G-lattices such that $\left[M_{1}\right]^{f l}=\left[M_{2}\right]^{f l}$. Then $\mathbb{F}\left(M_{2}\right)^{G} \approx \mathbb{F}\left(M_{2}\right)^{G}$ if one of the following conditions is satisfied:

(1) $M_{1}$ and $M_{2}$ are faithful $G$-lattices and $\mathbb{F}$ has a trivial $G$-action.

(2) $\mathbb{F} / \mathbb{F}^{G}$ is a $G$-Galois extension of fields.

\section{Flows in Graphs}

For the rest of this paper, we fix a field $\mathbb{F}$ of characteristic zero with trivial $G$-action.

Let $X=(V, E)$ be a (nonempty) finite graph (not necessarily simple). For a directed edge $e \in E$ we denote by $e_{S}, e_{T}$ the source and target of the edge. A flow on $X$ is a function $f: E \rightarrow \mathbb{Z}$ such 
that

$$
\sum_{\substack{e \in E \\ e_{S}=v}} f(e)=\sum_{\substack{e \in E \\ e_{T}=v}} f(e) \quad \text { for all } v \in V .
$$

The set of all flows is denoted by $F l(X)$ or $F l(V, E)$, which is of course an abelian group under the addition of functions. We will usually consider only connected graphs, where connected means that the underlying undirected graph is connected.

Another well known way to define this group comes from the homology groups of $X$. Let $\mathbb{Z}^{E}$, $\mathbb{Z}^{V}$ be the set of all integer valued functions on $E, V$ respectively and consider the following exact sequence

$$
\mathbb{Z}^{E} \stackrel{\partial_{E}}{\longrightarrow} \mathbb{Z}^{V} \stackrel{\varepsilon_{V}}{\longrightarrow} \mathbb{Z} \longrightarrow 0
$$

where

$$
\begin{aligned}
& \text { for all } f \in \mathbb{Z}^{E}: \partial_{E}(f)(v)=\sum_{e_{T}=v} f(e)-\sum_{e_{S}=v} f(e) \\
& \text { for all } h \in \mathbb{Z}^{V}: \quad \varepsilon_{V}(h)=\sum_{v \in V} h(v) .
\end{aligned}
$$

Let $I_{V}=\operatorname{ker}\left(\varepsilon_{V}\right)$. It is easily seen that $\operatorname{Im}\left(\partial_{E}\right) \subseteq I_{V}$ and the equality follows from connectedness of the graph. Finally, the group $F l(X)$ is nothing more than $\operatorname{ker}\left(\partial_{E}\right)$. Since $F l(X)$ is a subgroup of the f.g. free abelian group $\mathbb{Z}^{E}$, it is a f.g. free abelian group, and from the exact sequence above it has rank $|E|-|V|+1$.

Next we consider group actions on graphs.

Definition 4.1. Let $G$ be a finite group. We say that a $G$-action on $V, E$ is a graph action if the action on $E$ and $V$ is compatible, namely for every $g \in G$ and $e \in E$ we have that $g\left(e_{S}\right)=g(e)_{S}$ and $g\left(e_{T}\right)=g(e)_{T}$.

The action of $G$ on $V, E$ induce a $G$-action on $\mathbb{Z}^{V}, \mathbb{Z}^{E}$ respectively. Letting $\mathbb{Z}$ have a trivial $G$-action, we get that the sequence above is a sequence of $G$-modules. The induced action on $F l(X)=\operatorname{ker}\left(\partial_{E}\right)$ is the natural one, taking a flow $f$ and acting on it by the automorphisms of the graph. Finally, note that there is a natural isomorphism between $\mathbb{Z} V, \mathbb{Z} E$ and their duals $\mathbb{Z}^{V}, \mathbb{Z}^{E}$ respectively. We shall use these two presentations interchangeably.

The aim of this paper is to study the fields $\mathbb{F}(F l(X) \oplus \mathbb{Z} G)^{G}$ and to determine how close they are to being purely rational extensions of $\mathbb{F}$. The addition of $\mathbb{Z} G$ to $F l(X)$ is intended to make the lattice into a faithful $G$-lattice. If the action of $G$ on the $F l(X)$ is already faithful, then $\mathbb{F}(F l(X) \oplus \mathbb{Z} G)^{G}$ is rational over $\mathbb{F}(F l(X))^{G}$ by Theorem 3.4, thus in many cases this field is considered instead. In case the $G$-action on $F l(X)$ has nonzero kernel $K$, it is interesting to ask whether there is a connection between $\mathbb{F}(F l(X) \oplus \mathbb{Z} G)^{G}$ and $\mathbb{F}(F l(X))^{G / K}$ (see for example Subsection 5.2).

We note that while the entire investigation can be made using only the $G$-lattice notation without any reference to the flows in the graph, we have found that the use of flows have made some of the proofs much more intuitive, and in particular it helps in the later parts when we look for certain nice bases for the lattices $F l(X)$. The main idea is that if $f \in F l(V, E)$ is any flow such that $f(e)=1$ for some $e \in E$ and $g$ is any other flow, then $g-g(e) f$ is again a flow which is supported on $(V, E \backslash\{e\})$ (we redirected the flow on $e$ using $f$ ). Thus $F l(V, E) \cong \mathbb{Z} f \oplus F l(V, E \backslash\{e\})$ as abelian group. The next lemma generalizes this idea. 
Lemma 4.2. Let $X=(V, E)$ be a connected graph, $T=\left(V, E^{\prime}\right)$ a subgraph such that its underlying undirected graph is a tree, and write $E \backslash E^{\prime}=\left\{e_{1}, \ldots, e_{r}\right\}$. Let $f_{1}, \ldots, f_{r} \in F l(V, E)$ such that the matrix $\left(f_{i}\left(e_{j}\right)\right)$ is upper triangular with \pm 1 on the diagonal. Then $\left\{f_{1}, \ldots, f_{r}\right\}$ is a basis for $F l(V, E)$.

Proof. The proof is left to the reader.

Remark 4.3. Actually, the lemma above still holds whenever the matrix $\left(f_{i}\left(e_{j}\right)\right)$ is invertible, but we will only encounter triangular matrices in this paper.

The most natural choice of a set of edges is the complete directed graph. Let $E_{-}=\{(u, v) \mid u, v \in V$ distinct $\}$ and set $E_{+}=E_{-} \cup\{(v, v) \mid v \in V\}$. Clearly every flow in $F l\left(V, E_{+}\right)$can be decomposed as a flow supported on $E_{-}$and a flow supported on $E_{+} \backslash E_{-}$(self loops), or in other words $F l\left(V, E_{+}\right) \cong$ $F l\left(V, E_{+}\right) \oplus F l\left(V, E_{+} \backslash E_{-}\right)$. The set $E_{+} \backslash E_{-}$is just the self loops and $G$ acts on it as it acts on $V$, hence $F l\left(V, E_{+}\right) \cong F l\left(V, E_{-}\right) \oplus \mathbb{Z V}$. Thus, it is sufficient to consider the flows on the full directed graph without loops. The next lemma gives us a more general way to reduce the number of edges in the graph.

Lemma 4.4. Let $X=(V, E), X^{\prime}=\left(V, E^{\prime}\right)$ be two connected graphs with $G$-actions. Then $\mathbb{F}(F l(X) \oplus$ $\mathbb{Z} G)^{G}$ is stably isomorphic to $\mathbb{F}\left(F l\left(X^{\prime}\right) \oplus \mathbb{Z} G\right)^{G}$ over $\mathbb{F}$.

Proof. Consider the following exact diagram:

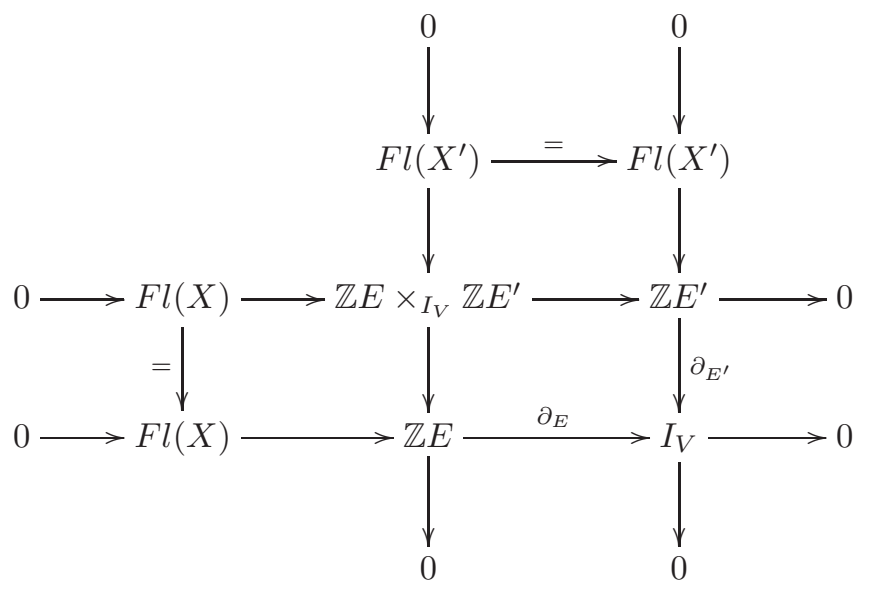

where $\mathbb{Z} E \times_{I_{V}} \mathbb{Z} E^{\prime}$ is the standard pullback. Since $\mathbb{Z} E, \mathbb{Z} E^{\prime}$ are permutation modules, it follows from Theorem 3.4 that $\mathbb{F}(\mathbb{Z} G \oplus F l(X))^{G} \approx_{\mathbb{F}} \mathbb{F}\left(\mathbb{Z} G \oplus F l\left(X^{\prime}\right)\right)^{G}$.

The previous lemma shows that up to stable rationality, the field $\mathbb{F}(F l(V, E) \oplus \mathbb{Z} G)^{G}$ is only a function of the set of vertices $V$ with its $G$-action. This result can also be motivated from the nature of flows on graphs. If $(V, E)$ is any connected $G$-graph and $\left(V, E^{\prime}\right)$ is a connected $G$-subgraph, then any flow going through an edge $e \in E \backslash E^{\prime}$, can be redirected to a flow in the graph $\left(V, E^{\prime}\right)$, since the vertices in $e$ are already connected in $E^{\prime}$. We can thus allow ourselves to write $F l(V)$ whenever the exact structure of $E$ is not important up to stable isomorphism of fields.

Next we reduce the number of vertices in $V$.

Lemma 4.5. Let $V=\bigsqcup V_{i}$ be the decomposition to $G$-orbits. Suppose that for some $i \neq j$ there is a $G$-equivariant map $\psi: V_{i} \rightarrow V_{j}$. Then $\mathbb{F}(F l(V) \oplus \mathbb{Z} G)^{G} \approx \mathbb{F}\left(F l\left(V^{\prime}\right) \oplus \mathbb{Z} G\right)^{G}$ where $V^{\prime}=V \backslash V_{i}$. 
Proof. Choose some $u \in V_{i}$ and set $v=\psi(u)$, then $\operatorname{stab}_{G}(u) \subseteq \operatorname{stab}_{G}(v)$. Let $T=\left\{t_{1}, \ldots, t_{k}\right\}$ be a set of left coset representatives of $\operatorname{stab}_{G}(u)$ in $G$. Let $E^{\prime}=\left\{\left(w_{1}, w_{2}\right) \mid w_{1}, w_{2} \in V^{\prime}\right\}$ so that $X^{\prime}=\left(V^{\prime}, E^{\prime}\right)$ is the complete (directed) graph on $V^{\prime}$ and set

$$
E=E^{\prime} \cup\left\{\left(t_{i} u, t_{i} v\right) \mid 1 \leq i \leq k\right\} .
$$

It is clear that $E^{\prime}$ is $G$-stable, and the stability of $E$ follows from the fact that $\operatorname{stab}_{G}(u) \subseteq \operatorname{stab}_{G}(v)$. The graph $X=(V, E)$ is just the full connected graph on $V^{\prime}$ plus a single edge from each vertex in $V_{i}$ to some vertex in $V_{j} \subseteq V \backslash V_{i}$. In particular, any flow in $X$ must be supported on $V \backslash V_{i}$, so that $F l(X)=F l\left(X^{\prime}\right)$ and the lemma follows.

The last lemma shows that we need only to consider such decompositions $V=\sqcup V_{i}$ where $\operatorname{Hom}\left(V_{i}, V_{j}\right)=\emptyset$ whenever $i \neq j$.

Note that if there is a map $V_{i} \rightarrow V_{j}$, then $\left|V_{j}\right|$ divides $\left|V_{i}\right|$. The opposite is also true if we have that $\left|V_{j}\right|=1$, and thus we have the following corollary.

Corollary 4.6. If $X=(V, E)$ is a connected $G$-graph which contains a vertex $v \in V$ with $\operatorname{stab}_{G}(v)=$ $G$, then $\mathbb{F}(F l(X) \oplus \mathbb{Z} G)^{G} \approx \mathbb{F}(\mathbb{Z} G)^{G}$.

The next generalization is when 1 is not one of the cardinalities of the $V_{i}$, but is their greatest common divisor. Assuming that $\operatorname{gcd}\left(\left|V_{1}\right|, \ldots,\left|V_{m}\right|\right)=1$, choose some $a_{i} \in \mathbb{Z}$ such that $\sum_{1}^{m} a_{i}\left|V_{i}\right|=1$.

For each $i$ choose some $v_{i} \in V_{i}$ and let $N_{i}=\sum_{v \in V_{i}} v \in \mathbb{Z} V$ be the norm element in $\mathbb{Z} V_{i}$. As before, we have the exact sequence

$$
0 \longrightarrow I_{V} \longrightarrow \bigoplus_{1}^{m} \mathbb{Z} V_{i} \stackrel{\varepsilon_{V}}{\longrightarrow} \mathbb{Z} \longrightarrow 0
$$

Define $\varphi: \mathbb{Z} \rightarrow \bigoplus_{1}^{m} \mathbb{Z} V_{i}$ by $\varphi(k)=\sum_{1}^{m} k a_{i} N_{i}$. This map is $G$-equivariant since the $N_{i}$ are $G$ invariant, and since $\varepsilon_{V}(\varphi(k))=k \sum_{1}^{m} a_{i}\left|V_{i}\right|=k$, the sequence splits.

If $E$ is any set of edges such that $X=(V, E)$ is a connected $G$-graph, then we have the following exact sequence

$$
0 \longrightarrow F l(X) \longrightarrow \mathbb{Z} \oplus \mathbb{Z} E \stackrel{i d \oplus \partial_{E}}{\longrightarrow} \mathbb{Z} \oplus I_{V} \cong \mathbb{Z} V \longrightarrow 0 .
$$

It follows that $F l(X)$ is quasi permutation and therefore

$$
\mathbb{F}(F l(X) \oplus \mathbb{Z} G)^{G} \approx \mathbb{F}(\mathbb{Z} \oplus \mathbb{Z} E \oplus \mathbb{Z} G)^{G} \approx \mathbb{F}(\mathbb{Z} G)^{G},
$$

thus reducing the problem to Noether's problem. As mentioned in Remark 3.5, the rationality of $\mathbb{F}(\mathbb{Z} G)^{G} / \mathbb{F}$ is known in many cases (for example if $G=S_{n}, G$ abelian and $\mathbb{F}$ contains enough roots of unity, etc).

\section{The CEnter of the Generic $G$-Crossed Product}

The second natural choice of graph is taking $V=G$, which as explained in the end of Section 2, corresponds (up to a stable isomorphism) to the center of the generic $G$-crossed product.

Let $E_{+}$be the set of all edges in the full directed graph on $V$ (including self loops). Fixing an identification of $V$ with $G$, define a degree function $\operatorname{deg}: E_{+} \rightarrow G$ by $\operatorname{deg}(g, h)=g^{-1} h$. For $S \subseteq G$ let $E_{+}(S)=\operatorname{deg}^{-1}(S)$ be all the edges of degree in $S$ and write $\operatorname{Cay}(G, S)=\left(V, E_{+}(S)\right)$ which is nothing more than the directed Cayley graph of $G$ corresponding to the set $S$. In particular $\operatorname{Cay}(G, S)$ is connected if and only if $S$ generates $G$. Excluding the self loops, let $G_{-}=(G \backslash\{e\})$, and note that $F l(G, G) \cong F l\left(G, G_{-}\right) \oplus \mathbb{Z} G$. 
As in the general case, if $(V, E),\left(V, E^{\prime}\right)$ are connected, then $\mathbb{F}(F l(V, E))^{G} \sim \mathbb{F}\left(F l\left(V, E^{\prime}\right)\right)^{G}$. In this case, where $G$ acts freely on $V$, a stronger result holds.

Lemma 5.1. Suppose that $G$ acts freely on $V$ (so that $V$ is a disjoint union of copies of $G$ ). Let $E^{\prime} \subseteq E$ be two $G$-sets of edges on $V$ such that $\left(V, E^{\prime}\right)$ is connected (and therefore also $(V, E)$ ). Then $F l(V, E) \cong F l\left(V, E^{\prime}\right) \oplus \mathbb{Z} G^{m}$ where $m=\frac{|E|-\left|E^{\prime}\right|}{|G|}$, and the isomorphism restricted to $F l\left(V, E^{\prime}\right)$ is the natural embedding.

Proof. Choose a set of orbit representatives $\left(v_{i}, w_{i}\right)$ of $E \backslash E^{\prime}$. For each $i$, choose some flow in $\left(V, E^{\prime}\right)$ from $w_{i}$ to $v_{i}$ (which exists since it is connected) and complete it with the edge $\left(v_{i}, w_{i}\right)$ to a circular flow $C_{i}$. By construction, each edge of $E \backslash E^{\prime}$ appears in exactly one of the flows $\bigcup_{i}\left\{g C_{i} \mid g \in G\right\}$. These can be used to redirect flows from edges in $E \backslash E^{\prime}$ into $E^{\prime}$, or more precisely $F l(V, E)=$ $F l\left(V, E^{\prime}\right) \oplus\left(\bigoplus_{i} \mathbb{Z}\left\{g C_{i} \mid g \in G\right\}\right)$ where for each $i$ we have $\mathbb{Z}\left\{g C_{i} \mid g \in G\right\} \cong \mathbb{Z} G$.

Corollary 5.2. Let $G$ be cyclic with generator $\sigma$. Then for any $S \subseteq G$ with $\sigma \in S$ we have $F l(G, S) \cong F l(G,\{\sigma\}) \oplus \mathbb{Z} G^{|S|-1}$ and $F l(G,\{\sigma\}) \cong \mathbb{Z}$ with the trivial action. In particular, $F l(G, S)$ is a permutation lattice.

Proof. The result follows since $\operatorname{Cay}(G,\{\sigma\})$ is just a simple cycle and $G$ acts trivially on $\operatorname{Fl}(G,\{\sigma\}) \cong$ $\mathbb{Z}$.

Remark 5.3. By Theorem 3.4, the field $\mathbb{F}\left(\mathbb{Z} \oplus(\mathbb{Z} G)^{k}\right)^{G}, k \geq 1$ is rational over $\mathbb{F}(\mathbb{Z} G)^{G}$. Fischer's theorem (see Remark 3.5) states that if $G$ is abelian with exponent $m$ and $\mathbb{F}$ contains a primitive $m$-th root of unity, then $\mathbb{F}(\mathbb{Z} G)^{G}$ is rational over $\mathbb{F}$. Thus, if $\mathbb{F}$ contains a primitive $|G|$-th root of unity for $G$ cyclic, then $\mathbb{F}(F l(V, E) \oplus \mathbb{Z} G)^{G}$ is rational over $\mathbb{F}$. For $\mathbb{F}=\mathbb{Q}$, even the extensions $\mathbb{Q}\left(\mathbb{Z} C_{n}\right)^{C_{n}} / \mathbb{Q}$ are not always rational, with the first counter example given by Swan in [35] for $n=47$ and the smallest counter example is $n=8$ [22].

As in many other cases, we will try to study the graphs $\operatorname{Cay}(G, S)$, where $\langle S\rangle=G$, by restricting the $G$-action to some subgroups. More generally, if $M$ is a $G$ lattice we denote by $M_{H}$ its restriction to the $H$-action where $H \leq G$. If $H$ is any subgroup of $G$ and $S_{0} \subseteq S$ is a generating set for $H$, then $\operatorname{Cay}\left(H, S_{0}\right)$ has a natural embedding in $\operatorname{Cay}(G, S)$. Thus, turning to their respective flow lattices, we see that $F l\left(H, S_{0}\right)$ is an $H$-sublattice of $F l(G, S)_{H}$ (the lattice with the $H$-action). The passage to this subgraph is given in the following lemma.

Lemma 5.4. Let $G$ be a group and $H$ a subgroup. Let $S$ be a generating set for $G$ containing a generating set $S_{0}$ for $H$. Then $F l(G, S) \cong F l\left(H, S_{0}\right) \oplus P$ as $H$ lattices where $P$ is a free $H$ lattice, and the restriction of the isomorphism to $F l\left(H, S_{0}\right)$ is the natural embedding.

Proof. This is just a fine tuning of Lemma 4.5 together with the previous lemma and is left to the reader.

In case $M_{1}, M_{2}$ are any two $G$-modules, the tensor product $M_{1} \otimes M_{2}$ is considered over $\mathbb{Z}$ with the diagonal $G$-action, namely $g\left(m_{1} \otimes m_{2}\right)=g m_{1} \otimes g m_{2}$ for any $g \in G$ and $m_{i} \in M_{i}, i=1,2$. If $M_{1}$ is a $\mathbb{Z} G-\mathbb{Z} H$ bimodule and $M_{2}$ an $H$-module for some subgroup $H \leq G$, then we write $M_{1} \otimes_{H} M_{2}$ for the $G$-module $M_{1} \otimes_{\mathbb{Z} H} M_{2}$ where $G$ acts through $M_{1}$. Under these notations we have the following.

Lemma 5.5. Let $G$ be a group, $H \leq G$ a subgroup and $M$ a $G$-lattice and set $M_{1}=\mathbb{Z} G / H \otimes M$ and $M_{2}=\mathbb{Z} G \otimes_{H} M_{H}$. Then the map $\varphi: M_{1} \rightarrow M_{2}$ defined by $\varphi(g H \otimes a)=g \otimes g^{-1} a$ is an isomorphism of $G$-lattices.

Proof. The proof is straight forward and is left to the reader. 
In the previous case we studied the action of $G$ on $\tilde{V}=\bigsqcup V_{i}$ where $\operatorname{gcd}\left(\left|V_{1}\right|, \ldots,\left|V_{k}\right|\right)=1$. Letting $H_{i}$ be the stabilizer of some $v_{i} \in V_{i}$ for each $i$, we see that $\operatorname{gcd}\left(\left[G: H_{1}\right], \ldots,\left[G: H_{k}\right]\right)=1$, since $V_{i} \cong G / H_{i}$ as $G$-sets and hence $\mathbb{Z} V_{i} \cong \mathbb{Z} G / H_{i}$. Clearly, if $M$ is any invertible $G$-lattice, namely a direct summand of a permutation $G$-lattice, it is also invertible as an $H$ lattice for any subgroup $H \leq G$. For the other direction, it is well known that if $M_{H_{i}}$ is invertible over subgroups $H_{i} \leq G$ that satisfy the condition above, then $M$ is invertible as a $G$-lattice. Let us recall the proof.

Consider the split exact sequence

$$
0 \longrightarrow I_{\tilde{V}} \longrightarrow \bigoplus_{1}^{k} \mathbb{Z} V_{i} \stackrel{\varepsilon_{\tilde{V}}}{\longrightarrow} \mathbb{Z} \longrightarrow 0
$$

Tensoring (over $\mathbb{Z}$ ) this sequence with the lattice $M$ we get a split exact sequence

$$
0 \longrightarrow I_{\tilde{V}} \otimes M \longrightarrow \bigoplus_{1}^{k}\left(\mathbb{Z}^{G} / H_{i} \otimes M\right) \stackrel{\varepsilon_{\tilde{V}}}{\longrightarrow} M \longrightarrow 0
$$

so that $M$ is a direct summand of $\bigoplus_{1}^{k}\left(\mathbb{Z} G / H_{i} \otimes_{\mathbb{Z}} M\right)$. By the previous lemma $\mathbb{Z} G / H \otimes M \cong \mathbb{Z} G \otimes_{H} M_{H}$, so the middle term in the sequence above is just $\bigoplus_{1}^{k}\left(\mathbb{Z} G \otimes_{H_{i}} M_{H_{i}}\right)$. It is now clear that if $M_{H_{i}}$ is a direct summand of a permutation $H_{i}$-lattice, then $M$ is a direct summand of a permutation $G$-lattice. By Lemma 5.1, the invertibility of $F l(G, S)$ doesn't depend on the generating set $S$, hence we can assume that we deal with $F l(G, G)$.

Corollary 5.6. Let $G$ be a group and $H_{i} \leq G$ subgroups such that $\operatorname{gcd}\left(\left[G: H_{1}\right], \ldots,\left[G: H_{k}\right]\right)=1$. The $G$-lattice $F l(G, G)$ is invertible if and only $\mathrm{Fl}\left(H_{i}, H_{i}\right)$ is invertible for each $i$.

A natural choice for $H_{i}$ in the above corollary are Sylow subgroups of $G$, and a trivial reason for $F l\left(H_{i}, H_{i}\right)$ to be invertible is if $H_{i}$ is cyclic. We are thus led to investigate groups such that all of their Sylow subgroups are cyclic, which are called $Z$-groups. These type of groups arise naturally in the theory of lattice invariant as we shall now recall.

Let $E$ be any set of edges on $V=G$ such that $X=(V, E)$ is connected. Recall that we have the exact sequences

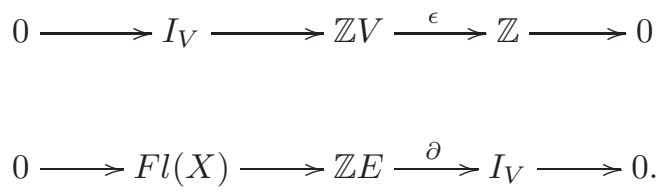

Since both $\mathbb{Z} V$ and $\mathbb{Z} E$ are free $G$ modules, it follows that they are also free over any subgroup $\tilde{G} \leq G$, and therefore $\hat{H}^{i}(\tilde{G}, \mathbb{Z} V)=\hat{H}^{i}(\tilde{G}, \mathbb{Z} E)=0$ for any $i \in \mathbb{Z}$. Taking the long exact sequence of the Tate cohomology we conclude that

$$
\hat{H}^{1}(\tilde{G}, F l(X)) \cong \hat{H}^{0}\left(\tilde{G}, I_{V}\right) \cong \hat{H}^{-1}(\tilde{G}, \mathbb{Z})=0,
$$

so that $F l(X)$ is coflasque. In particular, the second sequence above is a coflasque resolution of $I_{V}$. If $X^{\prime}=\left(V, E^{\prime}\right)$ is any other connected graph, it follows that $\left[F l\left(X^{\prime}\right)\right]=\left[I_{V}\right]^{\text {cofl }}=[F l(X)]$, thus there are permutation lattices $P, P^{\prime}$ such that $F l(X) \oplus P \cong F l\left(X^{\prime}\right) \oplus P^{\prime}$.

Taking $\left[I_{G}\right]^{c o f l}=\left[I_{V}\right]^{c o f l}=[F l(X)]$ and dualizing, we get that $\left[I_{G}^{*}\right]^{f l}=\left[I_{V}^{*}\right]^{f l}=[F l(X)]$. In [11], Endo and Miyata proved that there is an equality of sets coflasque $=$ flasque $=$ invertible for $G$-lattices if and only if $\left[I_{G}^{*}\right]^{f l}$ is invertible, which is of course equivalent to $F l(X)$ being invertible. Another equivalent condition given by Endo and Miyata is that $G$ is a $Z$-group, namely that every Sylow subgroup of $G$ is cyclic. 
Groups with cyclic Sylow subgroup also appear in Saltman's representing objects for crossed products in [30]. More precisely, let $\mathbb{L} / \mathbb{L}^{G}$ be a $G$-Galois extension and consider $F l\left(G, G_{-}\right)$. Saltman used Brauer theory in order to prove that the field $\mathbb{L}\left(F l\left(G, G_{-}\right)\right)^{G}$ (which is the center of Saltman's generic $G$-crossed product for the $\mathbb{L} / \mathbb{L}^{G}$ extension) is a retract rational extension of $\mathbb{L}^{G}$ if and only if $G$ is a $Z$-group if and only if there is an exact sequence

$$
0 \longrightarrow F l\left(G, G_{-}\right) \longrightarrow P \longrightarrow I \longrightarrow 0
$$

where $P$ is a permutation lattice and $I$ is invertible. Since $F l\left(G, G_{-}\right)$is coflasque, this last condition is also equivalent to $F l\left(G, G_{-}\right)$being invertible using Lemma 3.6.

On the other hand, suppose that $G$ is "far" from being a $Z$-group in the sense that it has some Sylow subgroup $P$ which is not a rank 1 or 2 abelian group. S Then Saltman proved that $\mathbb{F}(F l(X))^{G}$ is not retract rational over $\mathbb{F}$ and in particular it is not stably rational (Theorem 12.17 in [32]).

We note that here we consider the extension $\mathbb{F}(F l(X))^{G} / \mathbb{F}$ where $G$ acts on $\mathbb{F}$ trivially and not $\mathbb{L}(F l(X))^{G} / \mathbb{L}^{G}$ where $\mathbb{L} / \mathbb{L}^{G}$ is a $G$-Galois extension, so we cannot use directly the invertibility of $F l(X)$ in case $G$ is a $Z$-group. Nevertheless, we begin by investigating $Z$-groups.

5.1. Groups with cyclic $p$-Sylow subgroups. It is well known that each $Z$-group $G$ is isomorphic to a semidirect product $C_{n} \rtimes C_{m}$ where $C_{n}=\langle\sigma\rangle, C_{m}=\langle\tau\rangle$ are cyclic of coprime orders $n$ and $m$ respectively (see [17], section 9.4). As already seen, if $G$ is a $Z$-group, then $F l(G, E)$ is invertible. It follows that if $\mathbb{L} / \mathbb{L}^{G}$ is $G$-Galois, then $\mathbb{L}(M)^{G} / \mathbb{L}^{G}$ is a retract rational extension. A similar result holds in our case.

Lemma 5.7. Let $G$ be a Z-group. Let $\zeta$ be a primitive $t$-th root of unity, where $t$ is the highest power of 2 dividing $|G|$. If $\operatorname{Gal}(\mathbb{F}(\zeta) / \mathbb{F})$ is cyclic, then the field $\mathbb{F}(M)^{G}$ is retract rational over $\mathbb{F}$ for any invertible faithful G-lattice $M$.

Proof. Given any invertible lattice $M$ and a $G$-Galois extension $\mathbb{L} / \mathbb{L}^{G}$, Saltman showed that $\mathbb{L}(M)^{G} / \mathbb{L}^{G}$ is a retract rational extension ([30], Theorem 3.14). In addition, if $\operatorname{Gal}(\mathbb{F}(\zeta) / \mathbb{F})$ is cyclic and $G$ is a $Z$-group, he proved that for $\mathbb{L}=\mathbb{F}(\mathbb{Z} G)$ the extension $\mathbb{L}^{G} / \mathbb{F}$ is also retract rational (see [29], Theorems 2.1, 3.5, 5.3 where he proves that for such groups there is a generic Galois extension and has a lifting property, and in [30] Theorem 3.12 he proves that the lifting property implies retract rationality). In particular we get that $\mathbb{F} \subseteq \mathbb{F}(\mathbb{Z} G)^{G} \subseteq \mathbb{F}(\mathbb{Z} G \oplus M)^{G}$ is a tower of retract rational extensions, which by $[18]$ means that $\mathbb{F}(\mathbb{Z} G \oplus M)^{G} / \mathbb{F}$ is also a retract rational extension. Since $M$ is faithful and $\mathbb{Z} G$ is permutation, Proposition 3.3 shows that $\mathbb{F}(\mathbb{Z} G \oplus M)^{G} / \mathbb{F}(M)^{G}$ is rational and it follows that $\mathbb{F}(M)^{G} / \mathbb{F}$ is retract rational.

In particular, since $F l(G, E)$ is invertible, we conclude that $\mathbb{F}(F l(G, E) \oplus \mathbb{Z} G)^{G} / \mathbb{F}$ is retract rational, thus proving part (1) of Theorem 1.1.

Now that we know that $\mathbb{F}(F l(G))^{G} / \mathbb{F}$ is a retract rational extension given that $\mathbb{F}$ contains enough roots of unity, we turn to ask if it is a stably rational extension. One such result was given by Snider in [34]. Snider proved that if $G=\left\langle\sigma, \tau \mid \sigma^{n}=\tau^{2}=e, \tau \sigma \tau^{-1}=\sigma^{-1}\right\rangle$ is a dihedral group with $n$ odd and $\mathbb{F}$ contains a primitive $n$-th root of unity, then $\mathbb{F}(F l(G))^{G} / \mathbb{F}$ is stably rational.

As mentioned above, each $Z$-group $G$ is isomorphic to a semidirect product of cyclic groups of coprime orders $C_{n} \rtimes C_{m}$. Note that $C_{m}$ acts on $C_{n}$ by conjugation, and in the dihedral case the action is faithful. In what follows, we shall prove that we can always reduce the question to faithful actions.

Unless stated otherwise, for the rest of this section we fix the following notations. 
Let $G=\left\langle\sigma, \tau \mid \sigma^{n}=\tau^{m}=e, \tau^{-1} \sigma \tau=\sigma^{r}\right\rangle$ where $(n, m)=1$ and $r \in \mathbb{Z}$ satisfying $r^{m} \equiv_{n} 1$. Let $\langle\tau\rangle$ act on $\langle\sigma\rangle$ by conjugation and let $K=Z(G) \cap\langle\tau\rangle$ be the kernel of this action. Note that if $m_{0}$ is the order of $r$ in $\mathbb{Z} / n \mathbb{Z}$, then $K=\left\langle\tau^{m_{0}}\right\rangle$. It follows that $\tilde{G} \cong G / K=\left\langle\tilde{\sigma}, \tilde{\tau} \mid \tilde{\sigma}^{n}=\tilde{\tau}^{m_{0}}=e, \tilde{\tau} \tilde{\sigma} \tilde{\tau}^{-1}=\tilde{\sigma}^{r}\right\rangle$ and $\langle\tilde{\tau}\rangle$ acts faithfully on $\langle\tilde{\sigma}\rangle$. We also assume that $\sigma^{r} \neq \sigma$, and in particular $n>2$, since otherwise $G$ is cyclic. Finally, we let $M=F l(G,\{\sigma, \tau\})$. Our next goal is to show that we can move from $G$ to $\tilde{G}$ when computing the invariants.

As we have seen before, we have the split exact sequence

$$
0 \longrightarrow I \otimes M \longrightarrow(\mathbb{Z} G /\langle\sigma\rangle \otimes M) \oplus(\mathbb{Z} G /\langle\tau\rangle \otimes M) \stackrel{\varepsilon}{\longrightarrow} M \longrightarrow 0
$$

Let us investigate the projection $\varepsilon$ a little further.

From Lemma 5.4 and 5.5 we have the isomorphism

$$
\mathbb{Z} G /\langle\sigma\rangle \otimes M \cong \mathbb{Z} G \otimes_{\langle\sigma\rangle} M_{\langle\sigma\rangle} \cong \mathbb{Z} G \otimes_{\langle\sigma\rangle}\left(P_{1} \oplus F l(\langle\sigma\rangle,\langle\sigma\rangle)\right) \cong \mathbb{Z} G \otimes_{\langle\sigma\rangle}\left(P_{1} \oplus P_{2} \oplus \mathbb{Z}\right)
$$

where $P_{1}, P_{2}$ are free $\langle\sigma\rangle$-lattices. Let $\hat{S}=e \otimes 1$ in the right most term where $1 \in \mathbb{Z}$. The first and second isomorphisms from the right send it to $e \otimes S$ where $S$ is the flow on the cycle $e \rightarrow \sigma \rightarrow \sigma^{2} \rightarrow$ $\cdots \rightarrow \sigma^{n-1} \rightarrow e$ which is sent by the last isomorphism to $\langle\sigma\rangle \otimes S$.

Since $P_{1}, P_{2}$ are free $\langle\sigma\rangle$-lattices, we get that $\mathbb{Z} G \otimes\langle\sigma\rangle\left(P_{1} \oplus P_{2} \oplus \mathbb{Z}\right) \cong Q \oplus \mathbb{Z} G /\langle\sigma\rangle$ and $\hat{S}$ from above corresponds to $e\langle\sigma\rangle$ in $\mathbb{Z}^{G} /\langle\sigma\rangle$. By the previous argument we have that $\varepsilon(\hat{S})=S$. A similar argument is true for the subgroup $\langle\tau\rangle$. Putting it all together we get the split exact sequence

$$
0 \longrightarrow I \otimes M \longrightarrow P \oplus \mathbb{Z} G /\langle\sigma\rangle \oplus \mathbb{Z}^{G} /\langle\tau\rangle \stackrel{\varepsilon}{\longrightarrow} M \longrightarrow 0
$$

where $P$ is a $G$-free lattice. Additionally, denoting by $\hat{S}, \hat{T}$ the elements $e\langle\sigma\rangle, e\langle\tau\rangle$ in $\mathbb{Z} G /\langle\sigma\rangle, \mathbb{Z} G /\langle\tau\rangle$ respectively and letting $S, T$ be the flows $e \rightarrow \sigma \rightarrow \cdots \rightarrow \sigma^{n-1} \rightarrow e$ and $e \rightarrow \tau \rightarrow \cdots \rightarrow \tau^{m-1} \rightarrow e$ respectively, we get that $\varepsilon(\hat{S})=S$ and $\varepsilon(\hat{T})=T$. Our next step is to find another such exact sequence which is easier to work with.

Consider an exact sequence

$$
0 \longrightarrow \operatorname{ker}(\pi) \longrightarrow Q \oplus \mathbb{Z}^{G} /\langle\sigma\rangle \oplus \mathbb{Z} G /\langle\tau\rangle \stackrel{\pi}{\longrightarrow} M \longrightarrow 0
$$

where the restriction of $\pi$ to $\mathbb{Z}^{G} /\langle\sigma\rangle \oplus \mathbb{Z}^{G} /\langle\tau\rangle$ coincides with $\varepsilon$, and $Q$ is some free $G$-lattice. If $P^{\prime}$ is any free $G$-lattice, $H$ is any subgroup of $G$ and $i \in \mathbb{Z}$, then

$$
\hat{H}^{i}\left(P^{\prime} \oplus \mathbb{Z} G /\langle\sigma\rangle \oplus \mathbb{Z} G /\langle\tau\rangle\right) \cong \hat{H}^{i}(\mathbb{Z} G /\langle\sigma\rangle \oplus \mathbb{Z} G /\langle\tau\rangle),
$$

and since $\pi, \varepsilon$ coincide on $\mathbb{Z} G /\langle\sigma\rangle \oplus \mathbb{Z} G /\langle\tau\rangle$, it follows that their induced map on the Tate cohomology groups also coincide. The homomorphism $\varepsilon$ splits so its induced map in cohomology is always surjective, and therefore also $\pi$ 's induced maps. Finally, we have that

$$
\hat{H}^{0}\left(H, P^{\prime} \oplus \mathbb{Z}^{G} /\langle\sigma\rangle \oplus \mathbb{Z} G /\langle\tau\rangle\right) \longrightarrow \hat{H}^{0}(H, M) \longrightarrow \hat{H}^{1}(H, \operatorname{ker}(\pi)) \longrightarrow \hat{H}^{1}\left(P^{\prime} \oplus \mathbb{Z} G /\langle\sigma\rangle \oplus \mathbb{Z} G /\langle\tau\rangle\right)=0
$$

so that $\hat{H}^{1}(H, \operatorname{ker}(\pi))=0$ for all subgroups $H \leq G$, or equivalently it is a coflasque lattice. Since $M$ is invertible and $\operatorname{ker}(\pi)$ coflasque, we conclude from Lemma 3.6 that $\operatorname{ker}(\pi) \oplus M \cong \mathbb{Z} G \oplus \mathbb{Z} G /\langle\sigma\rangle \oplus \mathbb{Z} G /\langle\tau\rangle$ so that $\operatorname{ker}(\pi)$ is invertible and therefore $[M]^{f l}=[M]^{c o f l}=[\operatorname{ker}(\pi)]$. 
Let us consider the map $\pi$ defined as follows. Recall that $1 \leq r \leq n-1$ is the integer such that $\sigma \tau=\tau \sigma^{r}$. Define the flows $S, T, A$ to be

$$
\begin{aligned}
S & :=e \rightarrow \sigma \rightarrow \cdots \rightarrow \sigma^{n-1} \rightarrow e \\
T & :=e \rightarrow \tau \rightarrow \cdots \rightarrow \tau^{m-1} \rightarrow e \\
A & :=(e \rightarrow \sigma \rightarrow \sigma \tau)-\left(e \rightarrow \tau \rightarrow \tau \sigma \rightarrow \cdots \rightarrow \tau \sigma^{r}\right) .
\end{aligned}
$$

Clearly we have that $\sigma(S)=S$ and $\tau(T)=T$. Let $\hat{S}=e\langle\sigma\rangle, \hat{T}=e\langle\tau\rangle$ and $\hat{A}=e$ be generators for $\mathbb{Z} G /\langle\sigma\rangle, \mathbb{Z} G /\langle t\rangle$ and $\mathbb{Z} G$ respectively (as $\mathbb{Z} G$-modules), and define $\pi: \mathbb{Z} G /\langle\sigma\rangle \oplus \mathbb{Z} G /\langle\tau\rangle \oplus \mathbb{Z} G \rightarrow M$ by setting $\pi(\hat{S})=S, \pi(\hat{T})=T$ and $\pi(\hat{A})=A$. The maps $\pi$ and $\varepsilon$ coincide on $\mathbb{Z} G /\langle\sigma\rangle \oplus \mathbb{Z} G /\langle\tau\rangle$ so we are left to show that $\pi$ is surjective.

Denote by $N_{\sigma}, N_{\tau}$ the norm elements $N_{\sigma}=\sum_{i=0}^{n-1} \sigma^{i}$ and $N_{\tau}=\sum_{j=0}^{m-1} \tau^{j}$.

Lemma 5.8. The homomorphism $\pi: \mathbb{Z} G /\langle\sigma\rangle \oplus \mathbb{Z} G /\langle\tau\rangle \oplus \mathbb{Z} G \rightarrow M$ is surjective and $\operatorname{ker}(\pi)$ is spanned by the elements

$$
\begin{aligned}
V_{g} & =g\left(\sum_{j=0}^{m-1} \tau^{j} \sum_{i=0}^{r^{j}-1} \sigma^{i}(\hat{A})+\frac{r^{m}-1}{n} \cdot(\hat{S})+\hat{T}-\sigma(\hat{T})\right) \\
U_{\tau^{j}} & =\tau^{j}\left(N_{\sigma}(\hat{A})-\hat{S}+r \tau(\hat{S})\right)
\end{aligned}
$$

where $g \in G$ and $0 \leq j \leq m-1$.

Proof. For convenience, we sketch the proof for $S_{3} \cong C_{3} \rtimes C_{2}$. The general proof is a similar and is left to the reader.

The Cayley graph of $S_{3}$ with respect to $\{\sigma, \tau\}$ is

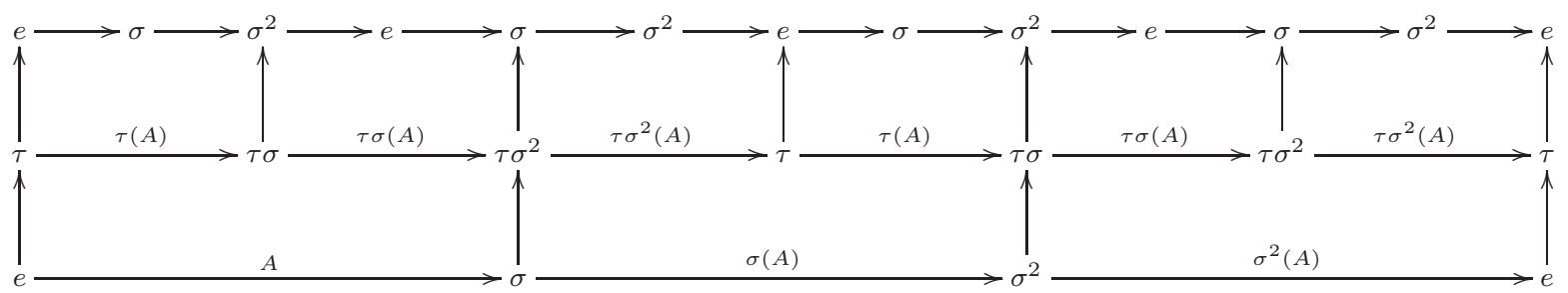

Note that some of the vertices and edges appear more than one time to make the illustration clearer.

By definition, $S, T$ and $A$ are in $\operatorname{Im}(\pi)$, so to show surjectivity it is enough to show that they generate $M$.

Suppose that $a \in M$ is a flow which contains the edge $\sigma^{2} \rightarrow \tau \sigma$ with coefficient $\lambda$. We can remove this edge by moving to $a-\lambda \sigma(A)$. Similarly we can remove the edge $\sigma \rightarrow \tau \sigma^{2}$ with the use of $A$, and without adding back the edge $\sigma^{2} \rightarrow \tau \sigma$. We can do the same trick in the top row and therefore we are left only with the edges of the form $\sigma^{i} \rightarrow \sigma^{i+1}, \tau \sigma^{i} \rightarrow \tau \sigma^{i+1}$ and $\tau^{j} \rightarrow \tau^{j+1}$. Clearly, this flow is a linear combination of $S, \tau(S)$ (horizontal lines) and $T$ (left vertical line), concluding that $a \in \operatorname{Im}(\pi)$.

We now turn to study the kernel $\operatorname{ker}(\pi)$. Clearly the norms $N_{\sigma}, N_{\tau}$ satisfy $N_{\sigma} \sigma=\sigma N_{\sigma}=N_{\sigma}$ and $N_{\tau} \tau=\tau N_{\tau}=N_{\tau}$. Since $\langle\sigma\rangle$ is normal in $G$, the norm $N_{\sigma}$ is central in $\mathbb{Z} G$.

By definition, the flow $A$ has +1 on the right and bottom edges and -1 on the left and top edges. We thus see that $A+\sigma(A)+\sigma^{2}(A)$ is zero on the edges of degree $\tau$ (which point up) and is making 
one cycle $e \rightarrow \sigma \rightarrow \sigma^{2} \rightarrow e$ on the bottom lines and twice the cycle $\tau \rightarrow \tau \sigma \rightarrow \tau \sigma^{2} \rightarrow \tau$ in the middle with a minus sign. In other words we have that $A+\sigma(A)+\sigma^{2}(A)=S-2 \tau(S)$, so that $U_{\tau^{0}} \in \operatorname{ker}(\pi)$. Similarly, the sum $A+\tau(A)+\tau \sigma(A)$ equals the square with the edges $e \rightarrow \sigma, \sigma(T)=\sigma \rightarrow \tau \sigma^{2} \rightarrow \sigma$ with plus sign and the edges $T=e \rightarrow \tau \rightarrow e$ and $e \rightarrow \sigma \rightarrow \sigma^{2} \rightarrow e \rightarrow \sigma$ with a minus sign. The top and the bottom edges gives us one cycle $e \rightarrow \sigma \rightarrow \sigma^{2} \rightarrow e$ (where $1=\frac{r^{m}-1}{n}$ for $r=m=2$ and $n=3)$. We conclude that $A+(\tau(A)+\tau \sigma(A))=\sigma(T)-T-S$ and therefore $V_{e} \in \operatorname{ker}(\pi)$.

Let $N=\operatorname{span}\left\{U_{g}, V_{\tau^{j}} \mid g \in G, 0 \leq j \leq m-1\right\}$. If $a \in \operatorname{ker}(\pi) / N$, then using the element $U_{\tau^{j}}$ and $V_{g}$ we can find a representative for it of the form

$$
x=\sum_{j=0}^{m-1}\left(\sum_{i=0}^{n-1} \alpha_{j, i} \tau^{j} \sigma^{i}(\hat{A})\right)+\beta \cdot \hat{T}+\gamma \cdot \hat{S}
$$

where $\alpha_{j}, \beta, \gamma \in \mathbb{Z}$. For any $0 \leq j \leq m-1$ and $1 \leq i \leq n-1$, the only elements in $\left\{\tau^{j_{1}} \sigma^{i_{1}} A\right\} \cup\{S, T\}$ that touch the edge $\left(\tau^{j} \sigma^{i}, \tau^{j} \sigma^{i} \tau\right)$ are $\tau^{j} \sigma^{i}(A)$ (with a minus sign) and $\tau^{j} \sigma^{i-1} A$ (with a plus sign). We conclude that $\alpha_{j, i}=\alpha_{j, i-1}$, so setting $\alpha_{j}=\alpha_{j, 0}$ we see that

$$
x=\sum_{j=0}^{m-1} \alpha_{j} \tau^{j} N_{\sigma}(\hat{A})+\beta \cdot \hat{T}+\gamma \cdot \hat{S} .
$$

Using the elements $U_{\tau^{j}}$ we can find another representative of the form

$$
x^{\prime}=\sum_{j=0}^{m-1} \alpha_{j} \tau^{j}(\hat{S}-r \tau \hat{S})+\beta \cdot \hat{T}+\gamma \cdot \hat{S}=\beta \hat{T}+\sum_{j=1}^{m-1}\left(\alpha_{j}-r \alpha_{j-1}\right) \tau^{j} \hat{S}+\left(\alpha_{0}-r \alpha_{m-1}+\gamma\right) \hat{S} .
$$

Since $\pi\left(x^{\prime}\right)=0$ and the elements $T,\left\{\tau^{j} S\right\}_{1}^{m}$ are linearly independent, $x^{\prime}$ must be zero and therefore $\operatorname{ker}(\pi)=N$.

We now turn to study the structure of $\operatorname{ker}(\pi)$. Recall that

$$
\begin{aligned}
V_{g} & =g\left(\sum_{j=0}^{m-1} \tau^{j} \sum_{i=0}^{r^{j}-1} \sigma^{i}(\hat{A})+\frac{r^{m}-1}{n} \cdot(\hat{S})+\hat{T}-\sigma(\hat{T})\right) \\
U_{\tau^{j}} & =\tau^{j}\left(N_{\sigma}(\hat{A})-\hat{S}+r \tau(\hat{S})\right) .
\end{aligned}
$$

The elements $\left\{\tau^{j} N_{\sigma}(\hat{A})\right\}_{j=0}^{m-1}$ are $\mathbb{Z}$ linearly independent and $U_{e}$ is $\sigma$ invariant, hence $M_{0}=\operatorname{span}_{\mathbb{Z}}\left\{U_{\tau^{j}}\right\}$ is a submodule of $\operatorname{ker}(\pi)$ isomorphic to $\mathbb{Z} G /\langle\sigma\rangle$. Obviously $M_{0} \subseteq \operatorname{ker}(\pi) \cap\langle\hat{A}, \hat{S}\rangle_{\mathbb{Z} G}$ and we wish to show that this is actually an equality.

Lemma 5.9. There is an equality $M_{0}=\operatorname{ker}(\pi) \cap\langle\hat{A}, \hat{S}\rangle_{\mathbb{Z} G}$ and $\operatorname{ker}(\pi) / M_{0} \cong I_{G /\langle\tau\rangle}$.

Proof. Let $x \in \operatorname{ker}(\pi) \cap\langle\hat{A}, \hat{S}\rangle_{\mathbb{Z} G}$. As in the previous lemma, the coefficients of $\tau^{j} \sigma^{i}(A)$ for $j$ fixed are the same, so $x$ has the form

$$
\sum_{0}^{m-1} \alpha_{j} \tau^{j} N_{\sigma} \hat{A}+\sum_{j=0}^{m-1} \beta_{j} \tau^{j} \hat{S} \equiv_{M_{0}} \sum_{0}^{m-1} \alpha_{j} \tau^{j}(\hat{S}-r \tau(\hat{S}))+\sum_{j=0}^{m-1} \beta_{j} \tau^{j} \hat{S}=\sum_{0}^{m-1}\left(\alpha_{j}-r \alpha_{j-1}+\beta_{j}\right) \tau^{j} \hat{S}
$$


where the subtraction in the indices is modulo $m$. Since $\pi(x)=0$ and $\left\{\tau^{j} S\right\}_{1}^{m}$ are linearly independent, we get that $x \equiv_{M_{0}} 0$, so $M_{0}=\operatorname{ker}(\pi) \cap\langle\hat{A}, \hat{S}\rangle_{\mathbb{Z} G}$. Since

$$
\operatorname{ker}(\pi) / M_{0} \leq \mathbb{Z} G \oplus \mathbb{Z} G /\langle\sigma\rangle \oplus \mathbb{Z} G /\langle\tau\rangle /\langle\hat{A}, \hat{S}\rangle \cong \mathbb{Z} G /\langle\tau\rangle
$$

and $\operatorname{ker}(\pi) / M_{0}$ is generated by the images $\{g(\hat{T}-\sigma(\hat{T}))\}_{g \in G}$ of $\left\{V_{g}\right\}_{g \in G}$, it follows that $\operatorname{ker}(\pi) / M_{0} \cong$ $I_{G /\langle\tau\rangle} \cdot$

Let $P$ be any permutation $G$-lattice with a surjection $\psi: P \rightarrow I_{G /\langle\tau\rangle}$. We have the following exact diagram

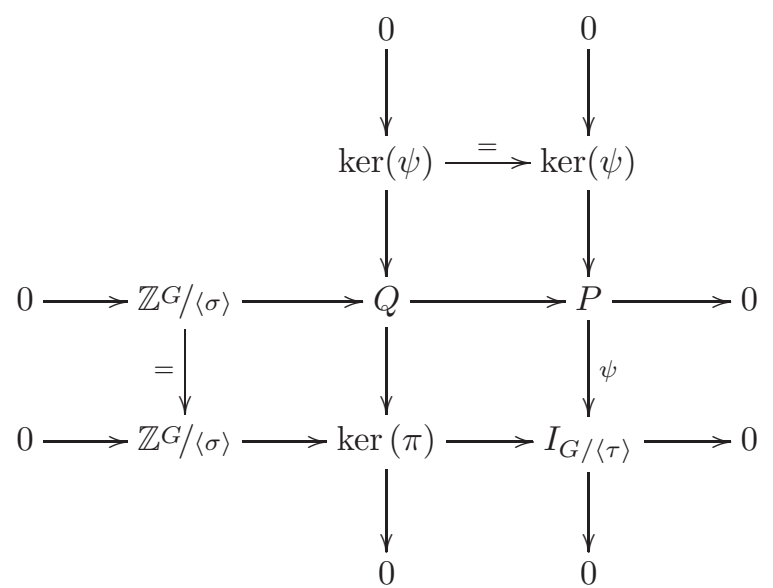

where $Q=\operatorname{ker}(\pi) \times_{I_{G /\langle\tau\rangle}} P$. Since $P$ and $\mathbb{Z} G /\langle\sigma\rangle$ are permutation lattices, the middle row splits by Lemma 3.6 and hence $Q$ is a permutation lattice as well. The lattice $\operatorname{ker}(\pi)$ is invertible so the middle column is a flasque resolution, hence $[M]^{f l}=[\operatorname{ker}(\pi)]=[\operatorname{ker}(\psi)]^{f l}$. On the other hand, the exact sequence on the right column corresponds to flows on the graph with edges $V=G /\langle\tau\rangle$. Using Theorem 3.8 we conclude the following.

Theorem 5.10. The field $\mathbb{F}(\mathbb{Z} G \oplus F l(G))^{G}$ is stably isomorphic to $\mathbb{F}(\mathbb{Z} G \oplus F l(G /\langle\tau\rangle))^{G}$ over $\mathbb{F}$.

While the action of $G$ on $\operatorname{Cay}(G, G)$ is faithful, its action on a graph with vertices $G /\langle\tau\rangle$ has kernel at least $Z(G) \cap\langle\tau\rangle$. As we shall see in the next section, this kernel can be mod out, namely that $\mathbb{F}(\mathbb{Z} G \oplus F l(G /\langle\tau\rangle))^{G}$ is stably isomorphic to $\mathbb{F}(\mathbb{Z} G /\langle\tau\rangle \oplus F l(G /\langle\tau\rangle))^{G /\langle\tau\rangle}$, thus proving part (2) of Theorem 1.1.

Remark 5.11. Let $G=\left\langle\sigma, \tau \mid \sigma^{n}=\tau^{2 m}=e, \tau \sigma \tau^{-1}=\sigma^{-1}\right\rangle$ with $(n, 2 m)=1$ so that $G / Z(G) \cap\langle\tau\rangle \cong$ $D_{2 n}$ is a dihedral group where $n$ is odd. Recall that Snider proved that if $\mathbb{F}$ contains a primitive $2 n$ root of unity, then $\mathbb{F}\left(D_{2 n}\right)^{D_{2 n}} / \mathbb{F}$ is stably rational, hence $\mathbb{F}(G)^{G} / \mathbb{F}$ is also stably rational. In [11], Endo and Miyata also proved that for such groups the lattice $I_{G}^{*}$ is a quasi-permutation lattice, which is equivalent to $F l(G, S)$ being stably permutation. In particular, this means that $\mathbb{F}(G)^{G}=$ $\mathbb{F}(F l(G, S) \oplus \mathbb{Z} G)^{G}$ is stably isomorphic to $\mathbb{F}(\mathbb{Z} G)^{G}$, so that $\mathbb{F}(\mathbb{Z} G)^{G} / \mathbb{F}$ is a stably rational extension.

5.2. Semidirect product of abelian groups. Let $G=N \rtimes H$ be a semidirect product of abelian groups. In this case we consider the set of edges $V=G / H$, with some fixed choice of copy of $H$ in $G$. The $G$-action on $V$ is not necessarily faithful. More precisely, if $h \in G$ is in the kernel, then it must 
be in $H$, and for every $g \in N$ we also have

$$
g H=h(g H)=h g h^{-1} H
$$

where $h g h^{-1} \in N$, so that $g=h g h^{-1}$. It follows that the kernel is exactly $H_{0}:=Z(G) \cap H$. Setting $\tilde{G}=G / H_{0}, \tilde{H}=H / H_{0}$, we see that the $G$-action on $V$ factors through $\tilde{G}$, and as such the structure of $V$ is $\tilde{V}=\tilde{G} / \tilde{H}$.

Let $E_{-}$be the set of all directed edges on $V \cong \tilde{V}$ which are not loops. The graphs $X=$ $\left(G / H, E_{-}(G)\right)$ and $\tilde{X}=\left(\tilde{G} / \tilde{H}, E_{-}(\tilde{G})\right)$ are isomorphic and the action of $G$ on $X \cong \tilde{X}$ is factored through $\tilde{G}$, and it follows that $\mathbb{F}(F l(X))^{G}=\left(\mathbb{F}(F l(X))^{H_{0}}\right)^{\tilde{G}}=\mathbb{F}(F l(\tilde{X}))^{\tilde{G}}$ under the identification of $X$ and $\tilde{X}$.

Recall that our usual setting is $\mathbb{F}(F l(X) \oplus \mathbb{Z} G)^{G}$ and we ask whether it is rational over $\mathbb{F}(F l(X))^{G}$. Writing $\mathbb{K}=\mathbb{F}(F l(X))$, so that $G$ act on $\mathbb{K}$ with kernel $H_{0}$, the question is the rationality of $\mathbb{K}(\mathbb{Z} G)^{G} / \mathbb{K}^{G}$. Two standard reasons used to show the rationality of such extensions are Proposition 3.3, where we need $G$ to act faithfully on $\mathbb{K}$, and Fischer's theorem where we need $G$ to be abelian of some exponent $m, \mathbb{K}^{G}=\mathbb{K}$ and that $\mathbb{K}$ contains a primitive root of unity of order $m$. While both of these conditions aren't true in this case, the next theorem shows how to combine them.

Lemma 5.12. Let $G=N \rtimes H$ be a semidirect product with $H$ abelian and set $H_{0}=Z(G) \cap H$ - the kernel of the $H$-action on $N$. Let $G$ act on a field $\mathbb{K}$ with kernel $H_{0}$, and assume that $\mathbb{K}^{G}$ contains a primitive root of unity of order $|H|$. Then $\mathbb{K}(\mathbb{Z} G / N)^{G}$ is rational over $\mathbb{K}^{G}$, and in particular $\mathbb{K}(\mathbb{Z} G)^{G}$ is stably rational over $\mathbb{K}^{G}$.

Proof. Note first that $G$ acts faithfully on $\mathbb{K}(\mathbb{Z} G / N)$ so

$$
\mathbb{K}(\mathbb{Z} G / N)^{G} \approx \mathbb{K}(\mathbb{Z} G / N \oplus \mathbb{Z} G)^{G} \approx \mathbb{K}(\mathbb{Z} G)^{G},
$$

hence it is enough to prove the rationality of $\mathbb{K}\left(\mathbb{Z}^{G} / N\right)^{G} / \mathbb{K}^{G}$.

Since $N$ acts trivially on $\mathbb{Z} G / N$ it follows that $\mathbb{K}\left(\mathbb{Z}^{G} / N\right)^{N}=\mathbb{K}^{N}\left(\mathbb{Z}^{G} / N\right)$ as $G / N \cong H$ field. We are thus reduced to the question of rationality of $\mathbb{K}(\mathbb{Z} H)^{H}$ over $\mathbb{K}^{H}$ where the kernel of the $H$ action on $\mathbb{K}$ is exactly $H_{0}$ and $\mathbb{K}^{H}$ contains an $m=|H|$ primitive root of unity $\zeta$.

Write $\mathbb{K}(\mathbb{Z} H)=\mathbb{K}\left(x_{h} \mid h \in H\right)$. Since $\mathbb{K}^{H}$ contains a root of unity of the order of the exponent of $H$, we get that $H^{*}:=\operatorname{Hom}\left(H, \mathbb{K}^{\times}\right)=\operatorname{Hom}\left(H,\left(\mathbb{K}^{H}\right)^{\times}\right)$. For any $\varphi \in H^{*}$ define $x_{\varphi}=\sum_{h} \varphi\left(h^{-1}\right) x_{h}$. We have that $\mathbb{K}\left(x_{h} \mid h \in H\right)=\mathbb{K}\left(x_{\varphi} \mid \varphi \in H^{*}\right)$ and $g\left(x_{\varphi}\right)=\varphi(g) x_{\varphi}$. Let $\psi: \prod_{|H|} \mathbb{Z} \rightarrow H_{0}^{*}$ be defined by

$$
\psi\left(k_{1}, \ldots, k_{m}\right)(h)=\prod \varphi_{i}^{k_{i}}(h) .
$$

Note that since $H$ is abelian and $\mathbb{K}$ contains a primitive $m$-th root of unity, the natural homomorphism $H^{*} \rightarrow H_{0}^{*}$ is surjective, and in particular $\psi$ is surjective. The kernel of $\psi$ is a subgroup of $\prod_{|H|} \mathbb{Z}$ of finite index $\left|H_{0}\right|$, so it is also free of rank $m$. If $\bar{k}=\left(k_{1}, \ldots, k_{m}\right)$ and $x^{(\bar{k})}=\prod x_{\varphi_{i}}^{k_{i}}$, then $g\left(x^{(\bar{k})}\right)=$ $\psi(\bar{k})(g) \cdot x^{(\bar{k})}$.

Let $\bar{k}^{(j)}=\left(k_{1}^{(j)}, \ldots, k_{m}^{(j)}\right) ; j=1, \ldots, m$ be a basis for $\operatorname{ker}(\psi)$ and set $x^{(j)}:=\prod x_{\varphi_{i}}^{k_{i}^{(j)}}$ for $1 \leq j \leq m$. By the previous argument we have that $\mathbb{K}\left(x^{(1)}, \ldots, x^{(m)}\right) \subseteq \mathbb{K}\left(x_{\varphi_{1}}, \ldots, x_{\varphi_{m}}\right)^{H_{0}}$. If $\left.\varphi_{i}\right|_{K}$ has order $t$ in $K^{*}$, then $x_{\varphi_{i}}^{t}$ is an invariant monomial, and therefore

$$
\left[\mathbb{K}\left(x^{(1)}, \ldots, x^{(m)}\right)\left[x_{\varphi_{i}}\right]: \mathbb{K}\left(x^{(1)}, \ldots, x^{(m)}\right)\right] \leq t .
$$


Using the decomposition of $H_{0}^{*}$ into cyclic groups and adding each of their generators, we get that

$$
\left|H_{0}\right|=\left[\mathbb{K}\left(x_{\varphi_{1}}, \ldots, x_{\varphi_{m}}\right): \mathbb{K}\left(x_{\varphi_{1}}, \ldots, x_{\varphi_{m}}\right)^{K}\right] \leq\left[\mathbb{K}\left(x_{\varphi_{1}}, \ldots, x_{\varphi_{m}}\right): \mathbb{K}\left(x^{(1)}, \ldots, x^{(m)}\right)\right] \leq\left|H_{0}\right|,
$$

and the equality $\mathbb{K}\left(x_{\varphi_{1}}, \ldots, x_{\varphi_{m}}\right)^{H_{0}}=\mathbb{K}\left(x^{(1)}, \ldots, x^{(m)}\right)$ follows (up to this point we followed Fischer's proof).

For each $1 \leq j \leq m$ and $h \in H / H_{0}$ we have $h\left(x^{(j)}\right)=\zeta_{h, j} x^{(j)}$ for elements (roots of unity) $\zeta_{h, j} \in \mathbb{K}$. For any fixed $j$, the map $h \mapsto \zeta_{h, j}$ is a 1-cocycle in $Z^{1}\left(H / H_{0}, \mathbb{K}^{\times}\right)$, which by Hilbert 90 has the form $\zeta_{h, j}=\frac{a_{j}}{h\left(a_{j}\right)}$ for some $a_{j} \in \mathbb{K}^{\times}$. It follows that $y^{(j)}=x^{(j)} a_{j}$ are $H / H_{0}$ invariant and algebraically independent. The proof is now finished by noting that

$$
\mathbb{K}\left(x^{(1)}, \ldots, x^{(m)}\right)^{\left(H / H_{0}\right)}=\mathbb{K}\left(y^{(1)}, \ldots, y^{(m)}\right)^{\left(H / H_{0}\right)}=\mathbb{K}^{\left(H / H_{0}\right)}\left(y^{(1)}, \ldots, y^{(m)}\right)=\mathbb{K}^{H}\left(y^{(1)}, \ldots, y^{(m)}\right) .
$$

Theorem 5.13. Let $G=N \rtimes H$ be a semidirect product of abelian groups and set $\tilde{G}=G / H \cap Z(G)$. Then setting $X=\left(G / H, E_{-}(G)\right)$ and $\tilde{X}=\left(\tilde{G} / \tilde{H}, E_{-}(\tilde{G})\right)$ we have $\mathbb{F}(F l(X) \oplus \mathbb{Z} G)^{G} \approx \mathbb{F}(F l(\tilde{X}) \oplus \mathbb{Z} \tilde{G})^{\tilde{G}}$.

Thus, by the theorem above we assume for the rest of this section that $H$ acts on $N$ faithfully. In particular, if the new group $G$ is dihedral $D_{2 n}$ with $n$ odd and $\mathbb{F}$ contains a primitive $2 n$ root of unity, then Theorem 5.10 together with Snider's result shows that $\mathbb{F}(F l(G /\langle\tau\rangle) \oplus \mathbb{Z} G)^{G} / \mathbb{F}$ is a stably rational extension.

5.3. $G=H \times K$ for groups $H, K$ of coprime orders. The aim of this section is to show that the field generated by $\mathbb{F}(F l(H) \oplus \mathbb{Z} H)^{H} \otimes \mathbb{F}(F l(K) \oplus \mathbb{Z} K)^{K}$ is stably isomorphic to $\mathbb{F}(F l(G) \oplus \mathbb{Z} G)^{G}$ whenever $G=H \times K$ such that $H$ and $K$ have coprime orders. In particular this means that in order to establish stable rationality for the group $G$, it is enough to prove it for $H$ and $K$.

Let $G$ be any finite group and consider its action on the graph $\operatorname{Cay}\left(G, G_{-}\right)=(V, E)$ and its corresponding lattices $\mathbb{Z} V, \mathbb{Z} E$ and $F l(V, E)$. A natural $\mathbb{Z}$-basis for $F l(V, E)$ is given by

$$
\begin{aligned}
d(g, h) & =(e \rightarrow g \rightarrow g h)-(e \rightarrow g h), g, h, g h \in G \backslash\{e\}, \\
d\left(g, g^{-1}\right) & =(e \rightarrow g)-(g \rightarrow e), g \in G \backslash\{e\} .
\end{aligned}
$$

In other words, we take the spanning tree consisting of all the edges $e \rightarrow g$ and for each edge not appearing in this tree we create a simple flow by adding to the edge a simple path from the tree. It is now easy to show that these flows satisfy the two cocycle condition, namely

$$
d\left(g_{1}, g_{2}\right)+d\left(g_{1} g_{2}, g_{3}\right)=d\left(g_{1}, g_{2} g_{3}\right)+g_{1}\left(d\left(g_{2}, g_{3}\right)\right),
$$

where we write $d(e, g)=d(g, e)=1$ for each $g \in G$. Furthermore, these relations generate all the other relations on this basis. This is not surprising since this is just the next part of the bar resolution of $G$ which starts with

$$
\mathbb{Z} E \stackrel{\partial}{\longrightarrow} \mathbb{Z} V \stackrel{\varepsilon}{\longrightarrow} \mathbb{Z} \longrightarrow 0 \text {. }
$$

Letting $d_{g}$ be the edge $e \rightarrow g$ for $g \neq e$ we get that flows $d(g, h)$ together with the edges $d_{g}$ constitute a basis for $\mathbb{Z} E$, and additionally we have

$$
d(h, g)=d_{h}+h\left(d_{g}\right)-d_{h g} .
$$

Note that applying $\partial$ on the equation above we get that $h\left(\tilde{d}_{g}\right)=\tilde{d}_{h g}-\tilde{d}_{h}$ which are the defining relation for the lattice $I_{G}$ (where we identify $\tilde{d}_{g}$ with $g-e$ ). Keeping all of this in mind, we have the following definitions (in multiplicative form). 
Let $\mathbb{L} / \mathbb{L}^{G}$ be a $G$-Galois extension of fields and $\alpha \in Z^{2}\left(G, \mathbb{L}^{\times}\right)$a 2-cocycle. Define $\mathbb{L}_{\alpha}\left(I_{G}\right)$ to be the field $\mathbb{L}\left(y_{g} \mid e \neq g \in G\right)$ where the $y_{g}$ are algebraically independent with the $G$-Galois action

$$
h\left(y_{g}\right)=\frac{y_{h g}}{y_{h}} \alpha(h, g),
$$

where we denote $y_{e}=1$. Note that the $G$-action can be rewritten as $\frac{y_{h} h\left(y_{g}\right)}{y_{h_{g}}}=\alpha(h, g)$.

For fixed elements $\lambda_{g} \in \mathbb{L}^{\times}, e \neq g \in G$, let $z_{g}=\lambda_{g} y_{g}$. Then clearly $\mathbb{L}_{\alpha}\left(I_{G}\right)=\mathbb{L}\left(z_{g} \mid e \neq g \in G\right)$ with the $G$-action defined by $\frac{z_{h} h\left(z_{g}\right)}{z_{h g}}=\frac{\lambda_{h} h\left(\lambda_{g}\right)}{\lambda_{h g}} \alpha(h, g)$, so in particular the field $\mathbb{L}_{\alpha}\left(I_{G}\right)$ is a function of the cohomology class of $\alpha$.

It can also be shown that $\mathbb{L}_{\alpha}\left(I_{G}\right)$ is a generic splitting field of $\Delta=\Delta\left(\mathbb{L} / \mathbb{L}^{G}, G, \alpha\right)$ in the sense of Amitsur [5]. We need only one property of such fields which we now prove.

Lemma 5.14. Let $\Delta=\Delta\left(\mathbb{L} / \mathbb{L}^{G}, G, \alpha\right)$ where $\Delta$ is split. Then $\mathbb{L}_{\alpha}\left(I_{G}\right)^{G}$ is stably rational over $\mathbb{L}^{G}$.

Proof. Since $\Delta$ splits, the cocycle $\alpha$ is cohomologous to 1 . As mentioned above, we may assume that the action is defined by $h\left(y_{g}\right)=\frac{y_{h g}}{y_{h}}$, or in other words, $\mathbb{L}_{\alpha}\left(I_{G}\right) \cong \mathbb{L}_{1}\left(I_{G}\right) \cong \mathbb{L}\left(I_{G}\right)$ where the correspondence is $g-e \leftrightarrow y_{g}$. The module $I_{G}$ is part of the exact sequence

$$
0 \longrightarrow I_{G} \longrightarrow \mathbb{Z} G \stackrel{\varepsilon}{\longrightarrow} \mathbb{Z} \longrightarrow 0,
$$

so by Theorem 3.4 we get that $\mathbb{L}(\mathbb{Z} G)^{G}$ is rational over $\mathbb{L}\left(I_{G}\right)^{G}$. On the other hand $\mathbb{L}(\mathbb{Z} G)^{G}$ is rational over $\mathbb{L}^{G}$ since $\mathbb{Z} G$ is a permutation lattice and $\mathbb{L} / \mathbb{L}^{G}$ is Galois, and the lemma follows.

We now turn to prove that if $\mathbb{F}(F l(H))^{H}$ and $\mathbb{F}(F l(K))^{K}$ are stably rational over $\mathbb{F}$ and $H, K$ are of coprime orders, then so is $\mathbb{F}(F l(G))^{G}$ for $G=H \times K$.

This was proved in the nongraded case by Katsylo [19], Schofield [33] and Saltman [31]. We will adapt Saltman's proof for the graded case.

Let $\mathbb{E}$ be a field with a $G$-action and consider the field $\mathbb{E}(F l(V, E))$. Letting $c(g, h)$ be the variables in $\mathbb{E}(F l(V, E))$ corresponding $d(g, h)$, it follows that $\{c(g, h) \mid e \neq g, h \in G\}$ are algebraically independent over $\mathbb{E}$ and $\sigma(c(g, h))=\frac{c(\sigma, g) c(\sigma g, h)}{c(\sigma, g h)}$ for every $\sigma, g, h \in G$, where we denote $c(g, e)=c(e, g)=1$ for all $g \in G$. We call such a field a generic $G$ 2-cocycle extension of $\mathbb{E}$, and denote it by $\mathbb{E}(c)$.

Lemma 5.15. Let $\mathbb{L} / \mathbb{L}^{G}$ be a $G$-Galois extension $\alpha \in Z^{2}\left(G, \mathbb{L}^{\times}\right)$and let $c$ be a generic $G$ 2-cocycle. Then we have the following:

(1) $\mathbb{L}(c)_{\alpha \cdot c}\left(I_{G}\right) \cong \mathbb{L}(c)_{c}\left(I_{G}\right)$.

(2) $\mathbb{L}(c)_{c}\left(I_{G}\right)^{G}$ is a rational extension of $\mathbb{L}^{G}$.

Proof. (1) The action on $\mathbb{L}_{\alpha c}\left(I_{G}\right)$ is defined by

$$
\frac{y_{h} h\left(y_{g}\right)}{y_{h g}}=\alpha(h, g) c(h, g) .
$$

Setting $c^{\prime}(h, g)=c(h, g) \alpha(h, g)$, we clearly get that $\left\{c^{\prime}(h, g) \mid e \neq h, g \in G\right\}$ are algebraically independent over $\mathbb{L}$, so $c^{\prime}$ is a generic $G$ 2-cocycle and $\mathbb{L}(c)_{\alpha \cdot c}\left(I_{G}\right) \cong \mathbb{L}\left(c^{\prime}\right)_{c^{\prime}}\left(I_{G}\right)$.

(2) As mentioned in the beginning of this section, $\mathbb{L}(c)_{c}\left(I_{G}\right)$ is just a complex way of writing $\mathbb{L}(\mathbb{Z} E)$. Since $\mathbb{L} / \mathbb{L}^{G}$ is $G$-Galois and $\mathbb{Z} E$ is a permutation lattice, we get that $\mathbb{L}(c)_{c}\left(I_{G}\right)^{G}=$ $\mathbb{L}(\mathbb{Z} E)^{G}$ is rational over $\mathbb{L}^{G}$. 
We now turn to decompose 2-cocycles on $G$ into two 2-cocycles on $H$ and $K$.

Let $c$ be a generic $G$ 2-cocycle and let $\alpha, \beta$ be inflations of generic $H \cong G / K$ and $K \cong G / H 2$ cocycles. More precisely, the elements $\left\{\alpha\left(h_{1}, h_{2}\right) \mid e \neq h_{1}, h_{2} \in H\right\}$ are algebraically independent, $\alpha\left(h_{1} g_{1}, h_{2} g_{2}\right)=\alpha\left(h_{1}, h_{2}\right)$ for any $h_{1}, h_{2} \in H$ and $g_{1}, g_{2} \in K$, and the $G$-action is defined by

$$
\begin{aligned}
g\left(\alpha\left(h_{1}, h_{2}\right)\right) & =\alpha\left(h_{1}, h_{2}\right) \quad \text { for all } g \in K, h_{1}, h_{2} \in H \\
h_{1}\left(\alpha\left(h_{2}, h_{3}\right)\right) & =\frac{\alpha\left(h_{1}, h_{2}\right) \alpha\left(h_{1} h_{2}, h_{3}\right)}{\alpha\left(h_{1}, h_{2} h_{3}\right)} \text { for all } h_{1}, h_{2}, h_{3} \in H
\end{aligned}
$$

and similarly define $\beta$.

We will show that $\mathbb{F}(\alpha, \beta, c)_{\alpha \beta c}\left(I_{G}\right)^{G}$ is rational over $\mathbb{F}(c)^{G}$ and over $\mathbb{F}(\alpha, \beta)^{G}$. The last field is the fraction field of $\mathbb{F}(\alpha)^{H} \otimes_{\mathbb{F}} \mathbb{F}(\beta)^{K}$, and this will finish the proof.

The second part follows from the previous two lemmas. More precisely we have $\mathbb{F}(\alpha, \beta, c)_{\alpha \beta c}\left(I_{G}\right)^{G}=$ $\mathbb{F}(\alpha, \beta)(c)_{\alpha \beta c}\left(I_{G}\right)^{G} \cong \mathbb{F}(\alpha, \beta)(c)_{c}\left(I_{G}\right)^{G}$ which is rational over $\mathbb{F}(\alpha, \beta)^{G}$.

Since $\alpha, \beta$ are two generic 2-cocycles for $H, K$ respectively, we expect that $\alpha \cdot \beta$ will have similar properties as a generic $G$ 2-cocycle. Indeed, the next lemma shows that the previous lemma still holds in this case.

Lemma 5.16. Let $\mathbb{L} / \mathbb{L}^{G}$ be a G-Galois extension where $G=H \times K$ with $(|H|,|K|)=1$. Let $\alpha, \beta$ be the inflations of the $H$ and $K$ generic 2-cocycles. Then the following holds:

(1) $\mathbb{L}(\alpha, \beta)_{\alpha \beta \gamma}\left(I_{G}\right) \cong \mathbb{L}(\alpha, \beta)_{\alpha \beta}\left(I_{G}\right)$ for all $\gamma \in Z^{2}\left(G, \mathbb{L}^{\times}\right)$.

(2) $\mathbb{L}(\alpha, \beta)_{\alpha \beta}\left(I_{G}\right)^{G}$ is a stably rational extension of $\mathbb{L}^{G}$.

Proof. (1) Since $H^{1}\left(K, \mathbb{L}^{\times}\right)=0$ by Hilbert 90 , we have the inflation restriction exact sequence

$$
0 \longrightarrow H^{2}\left(G / K,\left(\mathbb{L}^{\times}\right)^{K}\right) \stackrel{i n f}{\longrightarrow} H^{2}\left(G, \mathbb{L}^{\times}\right) \stackrel{r e s}{\longrightarrow} H^{2}\left(K, \mathbb{L}^{\times}\right)^{G / K}
$$

Since $|H|=|G / K|$ and $|K|$ are coprime, we can find $a, b \in \mathbb{Z}$ such that $a|G / K|+b|K|=1$. If $[\gamma] \in H^{2}\left(G, \mathbb{L}^{\times}\right)$is any 2-cocycle, then we can write $\gamma=\gamma_{H} \cdot \gamma_{K}$ where $\gamma_{H}=\gamma^{b|K|}$ and $\gamma_{K}=\gamma^{a|H|}$. The group $H^{2}\left(K, \mathbb{L}^{\times}\right)$is $|K|$-torsion, so res $\left(\gamma_{H}\right)=0$, and therefore $\gamma_{H} \in$ inf $\left(Z^{2}\left(G / K,\left(\mathbb{L}^{\times}\right)^{K}\right)\right.$. Switching the roles of $H$ and $K$ we get that $\gamma_{K} \in \inf \left(Z^{2}\left(G / H,\left(\mathbb{L}^{\times}\right)^{H}\right)\right.$. Letting $y_{g}$ be the indeterminates corresponding to $I_{G}$ in $\mathbb{L}(\alpha, \beta)_{\alpha \beta \gamma}\left(I_{G}\right)$, we have the action

$$
\begin{aligned}
h_{1} \tau_{1}\left(y_{h_{2} \tau_{2}}\right) & =\frac{y_{h_{1} h_{2} \tau_{1} \tau_{2}}}{y_{h_{1} \tau_{1}}} \alpha\left(h_{1} \tau_{1}, h_{2} \tau_{2}\right) \beta\left(h_{1} \tau_{1}, h_{2} \tau_{2}\right) \gamma\left(h_{1} \tau_{1}, h_{2} \tau_{2}\right) \\
& =\frac{y_{h_{1} h_{2} \tau_{1} \tau_{2}}}{y_{h_{1} \tau_{1}}} \alpha\left(h_{1}, h_{2}\right) \beta\left(\tau_{1}, \tau_{2}\right) \gamma_{H}\left(h_{1}, h_{2}\right) \gamma_{K}\left(\tau_{1}, \tau_{2}\right)
\end{aligned}
$$

for all $h_{1}, h_{2} \in H$ and $\tau_{1}, \tau_{2} \in K$. As in the previous lemma, defining $\tilde{\alpha}\left(g_{1}, g_{2}\right)=\alpha\left(g_{1}, g_{2}\right) \gamma_{H}\left(g_{1}, g_{2}\right)$ and $\tilde{\beta}\left(g_{1}, g_{2}\right)=\beta\left(g_{1}, g_{2}\right) \gamma_{K}\left(g_{1}, g_{2}\right)$ for $g_{1}, g_{2} \in G$, we get that $\tilde{\alpha}$ and $\tilde{\beta}$ are inflation of generic 2-cocycles in $H, K$ respectively, which show that $\mathbb{L}(\alpha, \beta)_{\alpha \beta \gamma}\left(I_{G}\right) \cong \mathbb{L}(\alpha, \beta)_{\alpha \beta}\left(I_{G}\right)$.

(2) Consider the field $\mathbb{K}=\mathbb{L}(\alpha, \beta)_{\alpha}\left(I_{G / K}\right)_{\beta}\left(I_{G / H}\right)_{\alpha \beta}\left(I_{G}\right)$, where the indeterminates of $I_{G / K}, I_{G / H}$ and $I_{G}$ are denoted by $z_{h}, w_{\tau}$ and $y_{g}$ respectively.

The 2-cocycle $\alpha \cdot \beta$ splits in $\mathbb{L}(\alpha, \beta)_{\alpha}\left(I_{G / K}\right)_{\beta}\left(I_{G / H}\right)$. Indeed, setting $\lambda_{h \tau}=z_{h} w_{\tau}$ for $h \in H$ and $\tau \in K$ we get that $\alpha \beta\left(g_{1}, g_{2}\right)=\frac{\lambda_{g_{1}} g_{1}\left(\lambda_{g_{2}}\right)}{\lambda_{g_{1} g_{2}}}$, where we use the fact that the $z_{h}$ are $K$ trivial and the $w_{\tau}$ are $H$-trivial. We conclude that $\mathbb{K} \cong \mathbb{L}(\alpha, \beta)_{\alpha}\left(I_{G / K}\right)_{\beta}\left(I_{G / H}\right)_{1}\left(I_{G}\right)$, so $\mathbb{K}^{G}$ is rational over $\mathbb{L}(\alpha, \beta)_{\alpha}\left(I_{G / K}\right)_{\beta}\left(I_{G / H}\right)^{G}$. Taking the invariants, first under $K$ and then under $H$, we see that the last field is rational over $\mathbb{L}^{G}$.

On the other hand, we have $\mathbb{K}=\mathbb{L}(\alpha, \beta)_{\alpha \beta}\left(I_{G}\right)_{\beta}\left(I_{G / H}\right)_{\alpha}\left(I_{G / K}\right)$, and we wish to show that 
$\alpha$ splits in $\mathbb{L}(\alpha, \beta)_{\alpha \beta}\left(I_{G}\right)^{K}$ so we can drop the last term $I_{G / K}$. Let $a, b \in \mathbb{Z}$ such that $|H| a+|K| b=1$, and set $\tilde{z}_{h}=z_{h} \cdot\left(\prod_{\tau \in K} \tau\left(y_{h}\right)\right)^{-b}$ for each $h \in H$ and note that $\tilde{z}_{h}$ is $K$ invariant. For $h, h^{\prime} \in H$ we get that

$$
\begin{aligned}
h^{\prime}\left(\tilde{z}_{h}\right) & =\left[\frac{z_{h^{\prime} h}}{z_{h^{\prime}}} \alpha\left(h^{\prime}, h\right)\right] /\left[\prod_{\tau} \tau\left(\frac{y_{h^{\prime} h}}{y_{h^{\prime}}} \alpha\left(h^{\prime}, h\right) \beta\left(h^{\prime}, h\right)\right)^{b}\right] \\
& =\left[\frac{z_{h^{\prime} h}}{z_{h^{\prime}}} \alpha\left(h^{\prime}, h\right)\right] /\left[\prod_{\tau} \tau\left(\frac{y_{h^{\prime} h}}{y_{h^{\prime}}}\right)^{a}\left(\alpha\left(h^{\prime}, h\right)\right)^{b|K|}\right]=\frac{\tilde{z}_{h^{\prime} h}}{\tilde{z}_{h^{\prime}}} \frac{\alpha\left(h^{\prime}, h\right)}{\alpha\left(h^{\prime}, h\right)^{b|K|}} \\
& =\frac{\tilde{z}_{h^{\prime} h}}{\tilde{z}_{h^{\prime}}} \alpha\left(h^{\prime}, h\right)^{a|H|} .
\end{aligned}
$$

Since $\alpha$ is an inflation of an $H$ 2-cocycle, we get that $\alpha^{a|H|} \sim 1$ where both cocycles are in $\mathbb{L}(\alpha, \beta)^{K}$. Thus, we can find $\lambda_{h} \in \mathbb{L}(\alpha, \beta)^{K}$ such that $\alpha\left(h_{1}, h_{2}\right)=\frac{\lambda_{h_{1}} h_{1}\left(\lambda_{h_{2}}\right)}{\lambda_{h_{1}} h_{2}}$ for all $h_{1}, h_{2} \in H$. Taking $\hat{z}_{h}=\tilde{z}_{h} \cdot \lambda_{h}$ we get that (1) $\hat{z}_{h}$ are $K$ invariant and (2) $H$ acts on them by $h^{\prime}\left(\hat{z}_{h}\right)=\frac{\hat{z}_{h^{\prime} h}}{\hat{z}_{h^{\prime}}}$, or in other words $\mathbb{K} \cong \mathbb{L}(\alpha, \beta)_{\alpha \beta}\left(I_{G}\right)_{\beta}\left(I_{G / H}\right)_{1}\left(I_{G / K}\right)$. By Lemma 5.14, we know that $\mathbb{K}^{H}$ is stably isomorphic to $\mathbb{K}=\mathbb{L}(\alpha, \beta)_{\alpha \beta}\left(I_{G}\right)_{\beta}\left(I_{G / H}\right)^{H}$, and actually it can be shown that this stably isomorphism uses $K$ invariant indeterminates, so we actually have that $\mathbb{K}^{G}$ is stably isomorphic to $\mathbb{L}(\alpha, \beta)_{\alpha \beta}\left(I_{G}\right)_{\beta}\left(I_{G / H}\right)^{G}$.

Repeating this process for $K$, we get that $\mathbb{K}^{G}$ is stably isomorphic to $\mathbb{L}(\alpha, \beta)_{\alpha \beta}\left(I_{G}\right)^{G}$ and we are done.

We now have all the ingredients for the proof of Theorem 1.3.

Proof. (of Theorem 1.3) Let $c$ be a generic $G$ 2-cocycle where $G=H \times K$ with $(|H|,|K|)=1$. Let $\alpha, \beta$ be the inflations of the $H$ and $K$ generic 2-cocycles. Consider the field $\mathbb{K}=\mathbb{F}(\alpha, \beta, c)_{\alpha \beta c}\left(I_{G}\right)^{G}$. From part (1) in Lemma 5.15 we get that

$$
\mathbb{F}(\alpha, \beta, c)_{\alpha \beta c}\left(I_{G}\right)=(\mathbb{F}(\alpha, \beta))(c)_{\alpha \beta c}\left(I_{G}\right) \cong(\mathbb{F}(\alpha, \beta))(c)_{c}\left(I_{G}\right)
$$

and from part $(2)$ we get that $(\mathbb{F}(\alpha, \beta))(c)_{c}\left(I_{G}\right)^{G}$ is rational over $\mathbb{F}(\alpha, \beta)^{G}$. Lemma 5.16 shows similarly that $\mathbb{F}(\alpha, \beta, c)_{\alpha \beta c}\left(I_{G}\right)^{G}$ is stably isomorphic to $\mathbb{F}(c)^{G}$.

Finally, we have

$$
\begin{aligned}
|G|=\left[\mathbb{F}(\alpha, \beta): \mathbb{F}(\alpha, \beta)^{G}\right] & \leq\left[\mathbb{F}(\alpha, \beta): \mathbb{F}(\alpha)^{H} \mathbb{F}(\beta)^{K}\right]=\left[\mathbb{F}(\alpha, \beta): \mathbb{F}(\alpha) \mathbb{F}(\beta)^{K}\right]\left[\mathbb{F}(\alpha) \mathbb{F}(\beta)^{K}: \mathbb{F}(\alpha)^{H} \mathbb{F}(\beta)^{K}\right] \\
& \leq\left[\mathbb{F}(\beta): \mathbb{F}(\beta)^{K}\right]\left[\mathbb{F}(\alpha): \mathbb{F}(\alpha)^{H}\right]=|K||H|=|G|,
\end{aligned}
$$

so there are equalities everywhere. In particular $\mathbb{F}(\alpha, \beta)^{G}=\mathbb{F}(\alpha)^{H} \cdot \mathbb{F}(\beta)^{K}$, which is isomorphic to the fraction field of $\mathbb{F}(\alpha)^{H} \otimes_{\mathbb{F}} \mathbb{F}(\beta)^{K}$ (since $\mathbb{F}(\alpha) \cap \mathbb{F}(\beta)=\mathbb{F}$ ). It follows that $\mathbb{F}(c)^{G}$ is stably isomorphic to the fraction field of $\mathbb{F}(\alpha)^{H} \otimes_{\mathbb{F}} \mathbb{F}(\beta)^{K}$.

\section{The Center of the generic Division Algebra}

The center of the generic division algebra of degree $n$ can also be represented using flows in graphs. More precisely, let $V$ be a set of vertices of cardinality $n$ and let $E_{-}=\{(v, u) \mid v, u \in V$ distinct $\}$. The graph $X=\left(V, E_{-}\right)$has a natural $S_{n}$-action, and the center of the generic division algebra of 
degree $n$ is $\mathbb{F}\left(F l(X) \oplus \mathbb{Z}^{S_{n} / S_{n-1}}\right)^{S_{n}}$ (up to stable isomorphism). Actually the part $\mathbb{Z}^{S_{n} / S_{n-1}}$ can be thought of as adding the self loops to $E_{-}$.

We finish this paper by recalling some of the results regarding the extension $\mathbb{F}\left(F l(X) \oplus \mathbb{Z} S_{n} / S_{n-1}\right)^{S_{n}} / \mathbb{F}$ and reinterpret them using the language of flows.

For $n=2$ the group $S_{n}=C_{2}$ is just the cyclic group of order 2 , so that $F l(X) \cong \mathbb{Z}$ because $X$ is just a cycle of order 2 (see also Corollary 5.2). The required field is thus $\mathbb{F}\left(\mathbb{Z} \oplus \mathbb{Z} C_{2}\right)^{C_{2}} \cong \mathbb{F}\left(\mathbb{Z} C_{2}\right)^{C_{2}}(x)$ which is rational over $\mathbb{F}$ since $\mathbb{F}\left(\mathbb{Z} C_{2}\right)^{C_{2}} / \mathbb{F}$ is rational.

For $n=3$ the group $S_{3}=D_{6}=\left\langle\sigma, \tau \mid \sigma^{3}=\tau^{2}=1, \tau \sigma \tau^{-1}=\sigma^{-1}\right\rangle$ and $V=G /\langle\tau\rangle$ as a $G$-set. Since all the Sylow subgroups of $S_{3}$ are cyclic, Theorem 1.1 and Corollary 1.2 show that $\mathbb{F}(X)^{S_{3}} / \mathbb{F}$ is stably rational (though we assume that $\mathbb{F}$ contains a primitive root of unity of order 3 ).

In case $n=p$ is prime, Saltman showed that the extension $\mathbb{F}(F l(X))^{S_{n}} / \mathbb{F}$ is retract rational [30]. Later on it was proved that $F l(X)$ is actually invertible (see Theorem 9.12 in [10] and section 3.1 in [8]). The retract rationality now follows since $\mathbb{F}\left(F l(X) \oplus \mathbb{Z} S_{n} / S_{n-1}\right) S_{n} / \mathbb{F}\left(\mathbb{Z} S_{n} / S_{n-1}\right)^{S_{n}}$ is a retract rational extension ([30], Theorem 3.14) and $\mathbb{F}\left(\mathbb{Z} S_{n} / S_{n-1}\right) S_{n} / \mathbb{F}$ is rational by the fundamental theorem of symmetric polynomials.

To prove that $F l(X)$ is invertible, recall that a $G$-lattice $M$ is invertible if and only if $M_{H_{i}}$, its restriction to the $H_{i}$-action, is invertible as an $H_{i}$-lattice for subgroups $H_{i}$ where $\operatorname{gcd}\left(\left[G: H_{1}\right], \ldots,\left[G: H_{k}\right]\right)=$ 1 (see the argument leading to Corollary 5.6). For $G=S_{n}$ choose $H_{1}=\langle(1,2,3, \ldots, n)\rangle \cong C_{n}$ and $H_{2}=\operatorname{stab}_{S_{n}}(\{n\}) \cong S_{n-1}$, hence for $n=p$ prime we get that $\left[G: H_{1}\right]=(p-1)$ ! and $\left[G: H_{2}\right]=p$ are coprime. The $H_{1}$-lattice $F l(X)_{H_{1}}$ is just $F l\left(C_{n}, C_{n}\right)$ which is a permutation lattice by Corollary 5.2 , since $H_{1}$ is cyclic. On the other hand, the $H_{2}$-lattice $F l(X)_{H_{2}}$ is again a flow in graph where there is a fixed vertex. We already showed in Corollary 4.6 that in such cases there is a reduction to Noether's problem $\mathbb{F}\left(\mathbb{Z} S_{n}\right)^{S_{n}} / \mathbb{F}$ which is known to be stably rational . A further investigation shows that $\mathrm{Fl}(\mathrm{X})_{\mathrm{H}_{2}}$ is already in itself a permutation lattice. Indeed, let $T$ be the subtree of $X$ consisting of the edges $\{(i, n) \mid 1 \leq i \leq n-1\}$. For each edge $e$ not in $T$ there is a unique way to complete it to a simple flow in the graph using the edges of $T$, which we denote by $C_{e}$. It is easily seen that $\left\{C_{e} \mid e \notin T\right\}$ is a basis for $F l(X)$ which is $H_{2}$-stable, so that $F l(X)$ is an $H_{2}$-permutation lattice.

Finally, in [7] Beneish proved the following. For $p$ prime, let $H \leq S_{p}$ be the subgroup generated by the cycle $(1,2,3, \ldots, p)$ and let $N$ be its normalizer. Then $N$ contains a cyclic group $C$ of order $p-1$ such that $N \cong H \rtimes C$ is isomorphic to the affine group of the finite field with $p$ elements. Beneish showed that under these notations the fields $\mathbb{C}(F l(X))$ is stably isomorphic to $\mathbb{C}\left(\mathbb{Z} S_{p} \otimes_{N} F l(X)\right)$ as $S_{n}$-fields (and therefore also as $N$-fields). Considering $F l(X)$ as a module over $\mathbb{Z} N$, we get the set of flows on the graph with vertices $N / C$, which is exactly the type of graphs appearing in Theorem 5.10 when we give a reduction for group with cyclic $p$-Sylow subgroups. Thus, a better understanding of these lattices over the affine group might lead to new results on the center of the standard generic division algebra.

\section{REFERENCES}

[1] E. Aljadeff and Y. Karasik, Polynomial identities and G-graded Azumaya algebras, Preprint .

[2] E. Aljadeff and Y. Karasik, Crossed products and their central polynomials, J. Pure Appl. Algebra 217(9):(2013), 1634-1641.

[3] E. Aljadeff and C. Kassel, Polynomial identities and noncommutative versal torsors, Adv. Math. 218(5):(2008), 1453-1495.

[4] S. A. Amitsur, On central division algebras, Israel J. Math. 12(4):(1972), 408-420. 
[5] S. A. Amitsur, Generic splitting fields, in F. M. J. v. Oystaeyen and A. H. M. J. Verschoren, editors, Brauer Groups in Ring Theory and Algebraic Geometry, number 917 in Lecture Notes in Mathematics, pages 1-24, Springer Berlin Heidelberg (1982).

[6] I. N. Balaba, Graded Prime PI-Algebras, J. Math. Sci. 128(6):(2005), 3345-3349.

[7] E. Beneish, Induction theorems on the stable rationality of the center of the ring of generic matrices, Trans. Amer. Math. Soc. 350(9):(1998), 3571-3585.

[8] C. Bessenrodt and L. Le Bruyn, Stable rationality of certain PGL_n-quotients, Invent. Math. 104(1):(1991), 179-199.

[9] S. Bloch, Torsion algebraic cycles, $K_{-} 2$, and Brauer groups of function fields, Bull. Amer. Math. Soc. (N.S.) 80(5):(1974), 941-945.

[10] J.-L. Colliot-ThÃ@iÃšne and J.-J. Sansuc, Principal homogeneous spaces under flasque tori: Applications, J. Algebra 106(1):(1987), 148-205.

[11] S. Endo and T. Miyata, On a classification of the function fields of algebraic tori, Nagoya Math J. 56:(1975), 85-104.

[12] B. Fein and M. Schacher, Brauer groups of rational function fields over global fields, in M. Kervaire and M. Ojanguren, editors, Groupe de Brauer, number 844 in Lecture Notes in Mathematics, pages 46-74, Springer Berlin Heidelberg (1981).

[13] E. Fischer, Die Isomorphie der Invariantenkorper der endlichen Abelschen Gruppen lineaerer Transformatioen, Nachrichten von der Gesellschaft der Wissenschaften zu Gottingen, Mathematisch-Physikalische Klasse pages $77-80$.

[14] E. Formanek, The center of the ring of 3x3 generic matrices, Linear and Multilinear Algebra 7(3):(1979), 203-212.

[15] E. Formanek, The center of the ring of $4 x 4$ generic matrices, J. Algebra 62(2):(1980), 304-319.

[16] E. Formanek, The Polynomial Identities and Invariants of n x n Matrices, American Mathematical Society (1992).

[17] M. Hall, The theory of groups, AMS Chelsea Pub., Providence, R.I. (1999).

[18] M.-c. Kang, Retract rational fields, J. Algebra 349(1):(2012), 22-37.

[19] P. I. Katsylo, Stable rationality of the field of invariants of linear representations of the groups PSL6 and PSL12, Mathematical notes of the Academy of Sciences of the USSR 48(2):(1990), 751-753.

[20] L. Le Bruyn, Permutation modules and rationality problems 1.

[21] L. Le Bruyn, Centers of generic division algebras, the rationality problem 1965-1990, Israel J. Math. 76(1):(1991), 97-111.

[22] H. W. Lenstra, Rational functions invariant under a finite abelian group, Invent. Math. 25(3):(1974), $299-325$.

[23] M. Lorenz, Multiplicative Invariant Theory, Springer, 2005 edition (2005).

[24] A. S. Merkurjev and A. A. Suslin, Cohomology of Severi-Brauer varieties and the norm residue homomorphism, Izvestiya Rossiiskoi Akademii Nauk. Seriya Matematicheskaya 46(5):(1982), 1011-1046.

[25] F. D. Meyer and E. Ingraham, Separable Algebras over Commutative Rings, Springer, 1971 edition (1971).

[26] M. Orzech and C. Small, The Brauer Group of Commutative Rings, M. Dekker, New York (1975).

[27] C. Procesi, Non-commutative affine rings, Atti Accad. Naz. Lincei Mem. Cl. Sci. Fis. Mat. Natur. Sez. I (8) 8:(1967), 237-255.

[28] S. Rosset, Group extensions and division algebras, J. Algebra 53(2):(1978), 297-303.

[29] D. J. Saltman, Generic Galois extensions and problems in field theory, Adv. Math. 43(3):(1982), 250-283.

[30] D. J. Saltman, Retract rational fields and cyclic Galois extensions, Israel J. Math. 47(2):(1984), 165-215.

[31] D. J. Saltman, A note on generic division algebras., Contemp. Math. 130:(1992), 385-394.

[32] D. J. Saltman, Lectures on Division Algebras, American Mathematical Society (1999).

[33] A. Schofield, Matrix invariants of composite size, J. Algebra 147(2):(1992), 345-349.

[34] R. Snider, Is the brauer group generated by cyclic algebras?, in D. Handelman and J. Lawrence, editors, Ring theory, volume 734 of Lecture Notes in Mathematics, pages 279-301, Springer Berlin / Heidelberg (1979).

[35] R. G. Swan, Invariant rational functions and a problem of Steenrod, Invent. Math. 7(2):(1969), 148-158.

[36] J. J. Sylvester, On the Involution of Two Matrices of the Second Order, The Collected Mathematical Paper 4.

Department of Mathematics, Technion-Israel Institute of Technology, Haifa 32000, Israel

E-mail address: eofirdav@tx.technion.ac.il 